\title{
On the multiple scales perturbation method for difference equations
}

\author{
W.T. van Horssen • M.C. ter Brake
}

Received: 19 December 2007 / Accepted: 15 May 2008 / Published online: 4 June 2008

(C) The Author(s) 2008. This article is published with open access at Springerlink.com

\begin{abstract}
In the classical multiple scales perturbation method for ordinary difference equations $(O \Delta E \mathrm{~s})$ as developed in 1977 by Hoppensteadt and Miranker, difference equations (describing the slow dynamics of the problem) are replaced at a certain moment in the perturbation procedure by ordinary differential equations $(O D E$ s). Taking into account the possibly different behavior of the solutions of an $O \Delta E$ and of the solutions of a nearby $O D E$, one cannot always be sure that the constructed approximations by the HoppensteadtMiranker method indeed reflect the behavior of the exact solutions of the $O \Delta E \mathrm{~s}$. For that reason, a version of the multiple scales perturbation method for $O \Delta E$ s will be presented and formulated in this paper completely in terms of difference equations. The goal of this paper is not only to present this method, but also to show how this method can be applied to regularly perturbed $O \Delta E \mathrm{~s}$ and to singularly perturbed, linear $O \Delta E \mathrm{~s}$.
\end{abstract}

Keywords Ordinary difference equation - Multiple scales perturbation method - Regularly and singularly perturbed problems · Asymptotic validity

\footnotetext{
W.T. van Horssen $(\bowtie) \cdot$ M.C. ter Brake

Delft Institute of Applied Mathematics (DIAM), Delft

University of Technology, Mekelweg 4, 2628 CD Delft,

The Netherlands

e-mail: W.T.vanHorssen@tudelft.nl

M.C. ter Brake

e-mail: M.C.terBrake@tudelft.nl
}

\section{Introduction}

Nowadays, the multiple time-scales perturbation method for differential equations is a well developed, well accepted, and a very popular method to approximate solutions of weakly nonlinear differential equations. This method was developed in the period 19351962 by Krylov and Bogoliubov, Kuzmak, Kevorkian, and Cole, Cochran, and Mahony. In the early 1970s, Nayfeh popularized this method by writing many papers and books on this subject (see for instance [20]). More recent books on this method and its historical development are, for instance, the books by Andrianov and Manevitch [3], Holmes [5], Kevorkian, and Cole [10], Murdock [18], and Verhulst [24]. The development of the multiple scales perturbation method for ordinary difference equations $(O \Delta E \mathrm{~s})$ started in 1960 with the work of Torng [23]. In this paper, a second order $O \Delta E$ is reduced to a system of two first order $O \Delta E$ s by means of the method of variation of parameters for $O \Delta E$ s. Then nonlinear terms are expanded in discrete Fourier series, and a KrylovBogoliubov method (or equivalently, an averaging method) is applied to obtain the equations that describe the slow dynamics of the problem approximately. A similar method was presented in 1970 by Huston in [7]. From the results in [7] and in [23], it is clear that the solution of a weakly perturbed (non) linear $O \Delta E$ behaves differently on different iteration scales. In 1977, Hoppensteadt and Miranker introduced in [6] the multiple scales perturbation method 
for $O \Delta E \mathrm{~s}$. For a problem with two significant iteration scales, these authors assume that the solution $x_{n}$ of an $O \Delta E$, for instance, depends on $n$ and $s=\epsilon n$ (that is, depends on a fast iteration scale and on a slow iteration scale). In the $O \Delta E, x_{n+1}$ is then replaced by $x(n+1, \epsilon(n+1))=x(n+1, s+\epsilon)$. In the perturbation scheme, $x(n+1, s+\epsilon)$ is expanded in a Taylor polynomial, that is, $x(n+1, s+\epsilon)=$ $x(n+1, s)+\epsilon \frac{\partial x}{\partial s}(n+1, s)+O\left(\epsilon^{2}\right)$, and to avoid unbounded terms (or secular terms) in the perturbation expansion for $x_{n}$, one finally has to solve ordinary differential equations (ODEs) due to the derivatives in the Taylor expansions. It should be remarked that in the perturbation procedure only for the description of the slow dynamics of the problem, the $O \Delta E$ s are replaced by $O D E$ s. A similar continuation approach was introduced in 1975 by Kovalev and Kosevich in [12] to study the vibrations of a one-dimensional anharmonic chain which is described by a system of $O D E$ s. In this paper [12], the slow dynamics of the problem leads to a partial differential equation (see also [11]). A slightly different multiple scales perturbation method for $O \Delta E$ s was introduced in [22] by Subramanian and Krishnan in 1979. In their approach, the difference operator $\Delta$ is replaced by partial difference operators. For a problem with two significant iteration scales, the authors of [22] introduced:

$$
\begin{aligned}
x_{n+1}-x_{n} & =\Delta x_{n}=\Delta x(n, s) \\
& =\Delta_{n}(n, s)+\epsilon \Delta_{s} x(n, s),
\end{aligned}
$$

where $\Delta_{n} x(n, s)=x(n+1, s)-x(n, s)$, and $\Delta_{s} x(n, s)=x(n, s+\epsilon)-x(n, s)$. This replacement is based on the two-timescales perturbation method for $O D E \mathrm{~s}$, where $x(t)$ is replaced by $\tilde{x}(t, \tau)$ with $\tau=\epsilon t$ and

$$
\frac{d x(t)}{d t}=\frac{\partial \tilde{x}(t, \tau)}{\partial t}+\epsilon \frac{\partial \tilde{x}(t, \tau)}{\partial \tau} .
$$

Nowadays, the method of Hoppensteadt and Miranker is assumed to be the standard form of the multiple scales perturbation method for $O \Delta E \mathrm{~s}$ (see for instance [5, 15-17]). Also recently, this method was "rediscovered" by Luongo [13] and by Maccari [14]. It should be observed, however, that many results concerning $O D E$ s carry over quite easily to corresponding results for $O \Delta E \mathrm{~s}$, while other results are completely different from their continuous counterparts. To illustrate some of these differences, the following examples can be considered.
Example 1 Consider the following $O D E$ for $x=x(t)$ :

$\frac{d x}{d t}=a x(1-x), \quad t>0$,

where $a$ is a positive constant, and $x(0)=x_{0}$. A forward Euler method is applied with positive stepsize $h$, and $x_{n}$ is an approximation of $x(t)$ at $t=n h$. The corresponding $O \Delta E$ becomes:

$x_{n+1}=x_{n}+a h x_{n}\left(1-x_{n}\right)$

for $n=0,1,2, \ldots$ Introducing $x_{n}=\frac{(1+a h)}{a h} y_{n}$, and $b=(1+a h)>0$, the following $O \Delta E$ for $y_{n}$ is obtained:

$y_{n+1}=b y_{n}\left(1-y_{n}\right)$.

For all $a>0$, the $O D E$ (2) has two equilibrium points (one stable point at $x=1$, and an unstable one at $x=0$ ), and the dynamics are simple. Whereas the logistic difference (3) is well known for its perioddoublings and its chaotic behavior for b-values between 3 and 4 . So, for certain parameter values, the solution of the $O D E$ and the solution of the (nearby) $O \Delta E$ behave quite differently.

Example 2 Consider the following $O D E$ for $x=x(t)$ :

$\epsilon \frac{d x}{d t}=-x, \quad t>0$,

where $\epsilon$ is a small, positive parameter, i.e., $0<\epsilon \ll 1$, and $x(0)=1$. The solution of the $O D E(4)$ is: $x(t)=$ $\exp \left(-\frac{t}{\epsilon}\right)$. When an explicit, forward Euler method is applied to (4) with positive stepsize $h$, and where $x_{n}$ is an approximation of $x(t)$ at $t=n h$, then the following $O \Delta E$ for $x_{n}$ will be obtained: (for $n=0,1,2, \ldots$, and $\left.x_{0}=1\right)$

$x_{n+1}=\left(1-\frac{h}{\epsilon}\right) x_{n} \Rightarrow x_{n}=\left(1-\frac{h}{\epsilon}\right)^{n}$.

For a fixed value of $h$ and for $\epsilon \downarrow 0 x_{n}$ obviously blows up, whereas $x(n h)$ rapidly tends to zero. When an implicit Euler method is applied to (4), then the following $O \Delta E$ for $x_{n}$ is obtained (again $h$ is a positive stepsize, $x_{n}$ is an approximation of $x(t)$ at $t=n h, n=$ $0,1,2, \ldots$, and $\left.x_{0}=1\right)$ :

$$
\begin{aligned}
& x_{n+1}-x_{n}=-\frac{h}{\epsilon} x_{n+1} \Rightarrow x_{n+1}=\frac{\epsilon}{h+\epsilon} x_{n} \Rightarrow \\
& x_{n}=\left(\frac{\epsilon}{h+\epsilon}\right)^{n} .
\end{aligned}
$$


For a fixed value of $h$ and for $\epsilon \downarrow 0 x_{n}$ behaves like $\left(\frac{\epsilon}{h}\right)^{n}=\exp \left(n \ln \left(\frac{\epsilon}{h}\right)\right)$, whereas $x(n h)$ behaves like $\exp \left(-\frac{n h}{\epsilon}\right)$. From the asymptotical point of view for $\epsilon \downarrow 0$, the behavior of $x_{n}$ and $x(n h)$ is quite different. When the trapezoidal formula is applied to (4), then the following $O \Delta E$ for $x_{n}$ is obtained (again $h$ is a positive stepsize, $x_{n}$ is an approximation of $x(t)$ at $t=n h, n=0,1,2, \ldots$, and $\left.x_{0}=1\right)$ :

$x_{n+1}-x_{n}=-\frac{h}{2 \epsilon}\left(x_{n+1}+x_{n}\right) \quad \Rightarrow$

$x_{n+1}=-\frac{(h-2 \epsilon)}{h+2 \epsilon} x_{n} \Rightarrow$

$x_{n}=(-1)^{n} \frac{(h-2 \epsilon)^{n}}{(h+2 \epsilon)^{n}}$.

Now, $x_{n}$ behaves like $(-1)^{n} \exp \left(n\left(\frac{-4 \epsilon}{h}+O\left(\epsilon^{3}\right)\right)\right)$, that is, $x_{n}$ is oscillatory (whereas $x(n h)$ is nonoscillatory), and the amplitudes of $x_{n}$ and $x(n h)$ behave differently for $\epsilon \downarrow 0$.

Example 3 Consider the following $O D E$ for $x=x(t)$ :

$\frac{d x}{d t}=\epsilon x^{2}, \quad t>0$,

where $\epsilon$ is a small, positive parameter, i.e., $0<\epsilon \ll 1$, and $x(0)=1$. The solution of this problem is: $x(t)=$ $(1-\epsilon t)^{-t}$. Obviously, the solution does not exist for $t \geq \epsilon^{-1}$. When an explicit, forward Euler method is applied to (5) with positive stepsize $h$, and where $x_{n}$ is an approximation of $x(t)$ at $t=n h$, then the following $O \Delta E$ for $x_{n}$ will be obtained: (for $n=0,1,2, \ldots$, and $\left.x_{0}=1\right)$

$x_{n+1}=x_{n}+\epsilon h x_{n}^{2}$.

For fixed values of $h$ and $\epsilon x_{n}$ obviously blows up, but not for $t=\epsilon^{-1}$ (but for $n \rightarrow \infty$ ). For instance, when $\epsilon=0.1$ and $h=0.01$, a simple computation shows that $x_{1000}$ (which is an approximation of $x(1)$ ) is equal to $193.1367 \ldots$ When an implicit Euler method is applied to (5), then the following $O \Delta E$ for $x_{n}$ is obtained (again $h, x_{n}, n$, and $x_{0}$ are defined as before):

$x_{n+1}=x_{n}+\epsilon h x_{n+1}^{2}$.

This quadratic equation in $x_{n+1}$ can be solved, yielding

$x_{n+1}=\frac{1}{2 \epsilon h} \pm \frac{1}{2 \epsilon h}\left(1-4 \epsilon h x_{n}\right)^{\frac{1}{2}}$.
Only the minus sign in (8) leads to $x_{n}$-values which approximate $x(t)$. For the plus sign in (8), $x_{n}$ becomes extremely large after one time-step. But also when the minus sign in (8) is used, $x_{n}$ will finally blow up, but not for $t=\epsilon^{-1}$ (but earlier). It should also be observed that for $x_{n} \geq(4 \epsilon h)^{-1}$ the $O \Delta E$ (8) is not defined. Finally, it should be remarked that when the classical Hoppensteadt-Miranker perturbation method for $O \Delta E \mathrm{~s}$ is applied to the $O \Delta E \mathrm{~s}(6)$ or (7), then for the slow dynamics of the solution the $O D E(5)$ is obtained, and one should be aware of the possibly different behavior near blow-up (in particular when blow-up occurs).

These examples clearly indicate that the solution of an $O D E$ and the solution of an (nearby) $O \Delta E$ can behave quite differently. The reader is referred to [1, $2,4,5,8,9,16]$ for some further striking differences (and similarities) in the theory for $O D E \mathrm{~s}$ and for $O \Delta E$ s. In the multiple scales perturbation method for $O \Delta E$ s as developed in [6] by Hoppensteadt and Miranker, difference equations (for the slow dynamics of the problem) are replaced at a certain moment by differential equations. Taking into account the possibly different behavior of the solutions of an $O \Delta E$ and of the solutions of an (nearby) $O D E$, one cannot always be sure that the constructed approximations by the Hoppensteadt-Miranker method indeed reflect the behavior of the exact solutions of the $O \Delta E$. For that reason, an improved version of the multiple scales perturbation method for $O \Delta E$ s will be presented and formulated in this paper completely in terms of difference equations.

The goal of this paper is not only to present this method, but also to show how this method can be applied to regularly perturbed $O \Delta E$ s and to singularly perturbed $O \Delta E$ s.

This paper is organized as follows. In Sect. 2 of this paper, the multiple scales perturbation method for $O \Delta E$ s will be presented completely in terms of difference operators. How this method can be applied to a second order regularly perturbed, weakly nonlinear $O \Delta E$ s will be shown in Sect. 3 of this paper. The asymptotic validity of the constructed approximations on sufficiently long iteration scales will be discussed in Sect. 4. How solutions of singularly perturbed, linear $O \Delta E$ s can be approximated will be shown in Sect. 5. Compared to the existing rescaling procedures 
for singularly perturbed $O D E \mathrm{~s}$ and $O \triangle E \mathrm{~s}$ (see, for instance, $[10,19,21,24])$ also a slightly revised rescaling procedure will be presented in Sect. 5 of this paper to find the significant scalings for some singularly perturbed, linear $O \Delta E$ s. Finally, in Sect. 6 of this paper, some conclusions will be drawn, and some remarks on future research will be made.

\section{The multiple scales perturbation method for $O \Delta E \mathbf{s}$}

In this section, the multiple scales perturbation method for $O \Delta E$ s will be presented in a complete "difference operator" setting. Before introducing this method, several operators have to be defined (and motivated). The well-known shift operator $E$, the difference operator $\Delta$, and the identity operator $I$ are defined as follows:

$E x_{n}=x_{n+1}, \quad \Delta x_{n}=x_{n+1}-x_{n}, \quad$ and $\quad I x_{n}=x_{n}$.

The relationship between these operators easily follows from (9):

$E=\Delta+I \quad \Leftrightarrow \quad \Delta=E-I$.

The solution of a weakly perturbed $O \Delta E$ usually contains a rapidly changing part in $n$, and a slowly changing part in $n$. This is usually referred to as multiple scales behavior. Consider the following functions:

$a_{n}=3^{n} \quad \Rightarrow$

$\Delta a_{n}=3^{n+1}-3^{n}=(3-1) 3^{n}=2 a_{n}=O\left(a_{n}\right)$,

$b_{n}=e^{\epsilon n} \quad \Rightarrow \quad \Delta b_{n}=e^{\epsilon(n+1)}-e^{\epsilon n}=O\left(\epsilon b_{n}\right)$,

$c_{n}=(1+\epsilon)^{n} \quad \Rightarrow$

$\Delta c_{n}=(1+\epsilon)^{n+1}-(1+\epsilon)^{n}=O\left(\epsilon c_{n}\right)$,

$d_{n}=3^{n}(1+\epsilon)^{n} \quad \Rightarrow$

$\Delta d_{n}=3^{n+1}(1+\epsilon)^{n+1}-3^{n}(1+\epsilon)^{n}=(2+3 \epsilon) d_{n}$.

From (11), it is obvious that $a_{n}$ only has a rapidly changing part in $n$, that $b_{n}$ and $c_{n}$ only have a slowly changing part in $n$, and that $d_{n}$ has a rapidly changing part in $n$ and a slowly one. To make this behavior more clear in notation, the following notations are proposed: $a_{n}=a(n), b_{n}=b(\epsilon n), c_{n}=c(\epsilon n)$, and $d_{n}=d(n, \epsilon n)$. It should be observed that these notations are similar to the ones used in the multiple timescales perturbation method for ODEs. Now it is assumed that $x_{n}=x(n, \epsilon n)$. This assumption implies that the solution of the $O \Delta E$ depends on two variables. So, the $O \Delta E$ actually becomes a partial difference equation. For that reason also, partial shift operators and partial difference operators have to be defined. The following definitions are proposed:

$$
\begin{aligned}
E_{1} x(n, \epsilon n) & =x(n+1, \epsilon n), \\
E_{\epsilon} x(n, \epsilon n) & =x(n, \epsilon(n+1)), \\
\Delta_{1} x(n, \epsilon n) & =x(n+1, \epsilon n)-x(n, \epsilon n) \\
& =\left(E_{1}-I\right) x(n, \epsilon n), \\
\Delta_{\epsilon} x(n, \epsilon n) & =x(n, \epsilon(n+1))-x(n, \epsilon n) \\
& =\left(E_{\epsilon}-I\right) x(n, \epsilon n) .
\end{aligned}
$$

From (9), (10), and (12), it follows that (assuming $\left.x_{n}=x(n, \epsilon n)\right)$ :

$$
\begin{aligned}
\Delta x_{n} & =x_{n+1}-x_{n}=x(n+1, \epsilon(n+1))-x(n, \epsilon n) \\
& =E_{1} E_{\epsilon} x(n, \epsilon n)-I x(n, \epsilon n) \\
& =\left(\Delta_{1}+I\right)\left(\Delta_{\epsilon}+I\right) x(n, \epsilon n)-I x(n, \epsilon n) \\
& =\left(\Delta_{1}+\Delta_{\epsilon}+\Delta_{1} \Delta_{\epsilon}\right) x(n, \epsilon n) .
\end{aligned}
$$

And so, it follows that

$\Delta=\Delta_{1}+\Delta_{\epsilon}+\Delta_{1} \Delta_{\epsilon}, \quad$ and $\quad E=E_{1} E_{\epsilon}$.

Furthermore, for the partial difference operators $\Delta_{1}$ and $\Delta_{\epsilon}$ it is assumed that (also based on (11)):

$\Delta_{1} x(n, \epsilon n)=O(x(n, \epsilon n)), \quad$ and

$\Delta_{\epsilon} x(n, \epsilon n)=O(\epsilon x(n, \epsilon n))$.

In fact, this assumption (14) implies that the variation in the dependent variable $x(n, \epsilon n)$ with respect to one of the independent variables is proportional to the product of the absolute value of the dependent variable and the variation in that particular independent variable. The examples as given by (11) are a motivation for the assumption (14).

From (13), it is obvious that in (1) the operator $\Delta_{1} \Delta_{\epsilon}$ is missing (see also [22]). When $x_{n}$ depends on $m+1$ scales, the given definitions can readily be 
generalized, yielding: (for $j=0,1, \ldots, m$ )

$x_{n}=x\left(n, \epsilon n, \epsilon^{2} n, \ldots, \epsilon^{m} n\right)$,

$E_{\epsilon^{j}} x\left(n, \ldots, \epsilon^{m} n\right)=x\left(n, \epsilon n, \ldots, \epsilon^{j}(n+1), \ldots, \epsilon^{m} n\right)$,

$\Delta_{\epsilon^{j}} x\left(n, \ldots, \epsilon^{m} n\right)=\left(E_{\epsilon^{j}}-I\right) x\left(n, \ldots, \epsilon^{m} n\right)$,

$E=E_{1} E_{\epsilon} E_{\epsilon^{2}} \cdots E_{\epsilon^{m}}$,

$\Delta=\left(\Delta_{1}+I\right)\left(\Delta_{\epsilon}+I\right) \ldots\left(\Delta_{\epsilon}+I\right)-I$,

$\Delta_{\epsilon^{j}} x\left(n, \ldots, \epsilon^{m} n\right)=O\left(\epsilon^{j} x\left(n, \ldots, \epsilon^{m} n\right)\right)$.

Now it will be shown how these operators can be used. For that reason, a simple example will be treated. Consider the weakly perturbed, linear, second order $O \Delta E$

$x_{n+2}+\epsilon x_{n+1}+x_{n}=0$,

where $\epsilon$ is a small parameter with $0<\epsilon \ll 1$. Using (9) and (10), it follows that (16) can be rewritten in:

$$
\begin{aligned}
& E^{2} x_{n}+\epsilon E x_{n}+I x_{n}=0 \Leftrightarrow \\
& (\Delta+I)^{2} x_{n}+\epsilon(\Delta+I) x_{n}+I x_{n}=0 \quad \Leftrightarrow \\
& \Delta^{2} x_{n}+(\epsilon+2) \Delta x_{n}+(2+\epsilon) x_{n}=0 .
\end{aligned}
$$

Assuming that $x_{n}$ depends on two scales (a fast scale $n$, and a slow scale $\epsilon n)$, it follows that $x_{n}=$ $x(n, \epsilon n)$ and that (16) or (17) becomes

$$
\begin{aligned}
& \left(\Delta_{1}+\Delta_{\epsilon}+\Delta_{1} \Delta_{\epsilon}\right)^{2} x(n, \epsilon n) \\
& \quad+(\epsilon+2)\left(\Delta_{1}+\Delta_{\epsilon}+\Delta_{1} \Delta_{\epsilon}\right) x(n, \epsilon n) \\
& \quad+(2+\epsilon) x(n, \epsilon n)=0 \Leftrightarrow \\
& \left(\Delta_{1}^{2}+2 \Delta_{1}+2\right) x(n, \epsilon n) \\
& \quad+\left(2 \Delta_{1}\left(\Delta_{\epsilon}+\Delta_{1} \Delta_{\epsilon}\right)\right. \\
& \quad+2\left(\Delta_{\epsilon}+\Delta_{1} \Delta_{\epsilon}\right) \\
& \left.\quad+\epsilon \Delta_{1}+\epsilon\right) x(n, \epsilon n)+O\left(\epsilon^{2} x(n, \epsilon n)\right)=0 \quad \Leftrightarrow \\
& \left(\Delta_{1}^{2}+2 \Delta_{1}+2\right) x(n, \epsilon n) \\
& \quad+\left(2\left(\Delta_{1}+I\right)\left(\Delta_{\epsilon}+\Delta_{1} \Delta_{\epsilon}\right)\right. \\
& \left.+\epsilon\left(\Delta_{1}+I\right)\right) x(n, \epsilon n) \\
& \quad+O\left(\epsilon^{2} x(n, \epsilon n)\right)=0 .
\end{aligned}
$$

To construct an approximation for $x_{n}=x(n, \epsilon n)$, one now has to substitute into (18) a formal power series (in $\epsilon$ ) for $x_{n}$, that is,

$$
x(n, \epsilon n)=x_{0}(n, \epsilon n)+\epsilon x_{1}(n, \epsilon n)+\epsilon^{2} x_{2}(n, \epsilon n)+\cdots .
$$

Then by taking together those terms of equal powers in $\epsilon$, one obtains as $O(1)$-problem

$$
\begin{aligned}
& \left(\Delta_{1}^{2}+2 \Delta_{1}+2\right) x_{0}(n, \epsilon n)=0 \\
& \quad \Leftrightarrow \quad x_{0}(n+2, \epsilon n)+x_{0}(n, \epsilon n)=0,
\end{aligned}
$$

and as $O(\epsilon)$-problem

$$
\begin{aligned}
& \epsilon\left(\Delta_{1}^{2}+2 \Delta_{1}+2\right) x_{1}(n, \epsilon n) \\
& \quad+\left(2\left(\Delta_{1}+I\right)\left(\Delta_{\epsilon}+\Delta_{1} \Delta_{\epsilon}+\frac{\epsilon}{2}\right)\right) x_{0}(n, \epsilon n)=0,
\end{aligned}
$$

and so on. The $O(1)$-problem (20) can readily be solved, yielding

$x_{0}(n, \epsilon n)=f_{0}(\epsilon n) \cos \left(\frac{n \pi}{2}\right)+g_{0}(\epsilon n) \sin \left(\frac{n \pi}{2}\right)$,

where $f_{0}(\epsilon n)$ and $g_{0}(\epsilon n)$ are still arbitrary functions, which can be used to avoid unbounded behaviour in $x_{1}(n, \epsilon n)$ on the $O\left(\frac{1}{\epsilon}\right)$ iteration scale.

The $O(\epsilon)$-problem (21) now becomes:

$$
\begin{aligned}
\epsilon & \left(x_{1}(n+2, \epsilon n)+x_{1}(n, \epsilon n)\right) \\
+ & 2\left(x_{0}(n+2, \epsilon(n+1))-x_{0}(n+2, \epsilon n)\right) \\
+ & \epsilon x_{0}(n+1, \epsilon n)=0 \Leftrightarrow \\
\epsilon( & \left.x_{1}(n+2, \epsilon n)+x_{1}(n, \epsilon n)\right) \\
= & \left(2 \Delta_{\epsilon} f_{0}(\epsilon n)-\epsilon g_{0}(\epsilon n)\right) \cos \left(\frac{n \pi}{2}\right) \\
& +\left(2 \Delta_{\epsilon} g_{0}(\epsilon n)+\epsilon f_{0}(\epsilon n)\right) \sin \left(\frac{n \pi}{2}\right) .
\end{aligned}
$$

In the $O \Delta E$ (23) for $x_{1}(n, \epsilon n)$, it is obvious that the right-hand side contains terms (i.e., $\cos \left(\frac{n \pi}{2}\right)$ and $\sin \left(\frac{n \pi}{2}\right)$ ), which are solutions of the homogeneous $O \Delta E$. Then to avoid unbounded or secular behavior 
in $x_{1}(n, \epsilon n)$, it follows that $f_{0}(\epsilon n)$ and $g_{0}(\epsilon n)$ have to satisfy:

$2 \Delta_{\epsilon} f_{0}(\epsilon n)-\epsilon g_{0}(\epsilon n)=0$,

$2 \Delta_{\epsilon} g_{0}(\epsilon n)+\epsilon f_{0}(\epsilon n)=0$.

System (24) for $f_{0}(\epsilon n)$ and $g_{0}(\epsilon n)$ can readily be solved (see, for instance, [4, Chap. 3]), yielding

$$
\begin{aligned}
f_{0}(\epsilon n)= & a_{0}\left(1+\frac{\epsilon^{2}}{4}\right)^{\frac{n}{2}} \cos (n \mu(\epsilon)) \\
& +b_{0}\left(1+\frac{\epsilon^{2}}{4}\right)^{\frac{n}{2}} \sin (n \mu(\epsilon)), \\
g_{0}(\epsilon n)= & -a_{0}\left(1+\frac{\epsilon^{2}}{4}\right)^{\frac{n}{2}} \sin (n \mu(\epsilon)) \\
& +b_{0}\left(1+\frac{\epsilon^{2}}{4}\right)^{\frac{n}{2}} \cos (n \mu(\epsilon)),
\end{aligned}
$$

where $a_{0}$ and $b_{0}$ are arbitrary constants, and where $\mu(\epsilon)$ is given by $\cos (\mu(\epsilon))=\left(1+\frac{\epsilon^{2}}{4}\right)^{-\frac{1}{2}}$, and $\sin (\mu(\epsilon))=\frac{\epsilon}{2}\left(1+\frac{\epsilon^{2}}{4}\right)^{-\frac{1}{2}}$. From these expressions, $\mu(\epsilon)$ can be approximated by

$\mu(\epsilon)=\frac{1}{2} \epsilon-\frac{1}{24} \epsilon^{3}+O\left(\epsilon^{5}\right)$,

and from (23), $x_{1}(n, \epsilon n)$ can be determined, yielding

$x_{1}(n, \epsilon n)=f_{1}(\epsilon n) \cos \left(\frac{n \pi}{2}\right)+g_{1}(\epsilon n) \sin \left(\frac{n \pi}{2}\right)$,

where $f_{1}(\epsilon n)$ and $g_{1}(\epsilon n)$ are still arbitrary functions which can be used to avoid secular terms in $x_{2}(n, \epsilon n)$. At this moment, however, we are not interested in the higher order approximations. For that reason, we will take in (27) $f_{1}(\epsilon n)$ and $g_{1}(\epsilon n)$ equal to the constants $a_{1}$ and $b_{1}$, respectively. So far, we have constructed an approximation for the solution of the $O \Delta E$ (16). In this case, the approximation $x_{0}(n, \epsilon n)$ can be compared with the exact solution of the $O \Delta E$ (16). The exact solution is given by

$x_{n}=a \cos (n \theta(\epsilon))+b \sin (n \theta(\epsilon))$,

where $a$ and $b$ are arbitrary constants, and where $\theta(\epsilon)$ is given by $\cos (\theta(\epsilon))=-\frac{\epsilon}{2}$ and $\sin (\theta(\epsilon))=$ $\left(1-\frac{\epsilon^{2}}{4}\right)^{\frac{1}{2}}$, and $\theta(\epsilon)$ can be approximated by $\theta(\epsilon)=$
$\frac{\pi}{2}+\frac{\epsilon}{2}+\frac{\epsilon^{3}}{48}+O\left(\epsilon^{5}\right)$. The approximation $x_{0}(n, \epsilon n)$ is given by (22), (25), and (26). This approximation can be rewritten in the following form

$$
\begin{aligned}
x_{0}(n, \epsilon n)= & a_{0}\left(1+\frac{\epsilon^{2}}{4}\right)^{\frac{n}{2}} \cos \left(\frac{n \pi}{2}+n \mu(\epsilon)\right) \\
& +b_{0}\left(1+\frac{\epsilon^{2}}{4}\right)^{\frac{n}{2}} \sin \left(\frac{n \pi}{2}+n \mu(\epsilon)\right) .
\end{aligned}
$$

From (28) and (29), it can readily be deduced that the difference between the exact solution $x_{n}$ and the approximation $x_{0}(n, \epsilon n)$ is of order $\epsilon$ for $n \sim \frac{1}{\epsilon}$. So, the constructed approximation is $O(\epsilon)$ accurate on an iteration scale of order $\frac{1}{\epsilon}$. Usually, of course, the exact solution of a weakly (non)linearly perturbed $O \Delta E$ will not be available. In Sect. 4 of this paper, it will be shown how for such cases the asymptotic validity of an approximation can be obtained on a sufficiently long iteration scale. In the next section of this paper, it will be shown how the multiple scales perturbation method can be applied to a second order, weakly nonlinear, regularly perturbed $O \Delta E$.

\section{On a weakly nonlinear, regularly perturbed $O \Delta E$}

In this section, an approximation of the solution of a second order, weakly nonlinear, regularly perturbed $O \Delta E$ with a Van der Pol type of nonlinearity will be constructed. The $O \Delta E$ can be obtained by using a central finite difference approximation of the continuous Van der Pol equation. The $O \Delta E$ is given by

$x_{n+2}-2 \cos (\theta) x_{n+1}+x_{n}=\epsilon\left(1-x_{n+1}^{2}\right)\left(x_{n+2}-x_{n}\right)$,

where $\epsilon$ is a small parameter, that is, $0<\epsilon \ll 1$, and where $\theta$ is constant (which is related to the stepsize in making the continuous van der Pol equation discrete). Other studies of (30) or similar equations as discretizations of the continuous van der Pol differential equation or similar differential equations can be found in $[5,6,13,14,16,17,22]$. It turns out that a straightforward, naive perturbation expansion for $x_{n}$ (that is, $\left.x_{0}(n)+\epsilon x_{1}(n)+\epsilon^{2} x_{2}(n)+\cdots\right)$ will lead to secular 
behavior in the expansion. To avoid this, a two scales perturbation method will be used with $x_{n}=x(n, \epsilon n)$. To apply the perturbation method, the $O \Delta E$ (30) first has to be rewritten with difference operators (see also (9), (10)), yielding

$$
\begin{aligned}
& \Delta^{2} x_{n}+(2-2 \cos (\theta))\left(\Delta x_{n}+x_{n}\right) \\
& \quad=\epsilon\left(1-\left(\Delta x_{n}+x_{n}\right)^{2}\right)\left(\Delta^{2} x_{n}+2 \Delta x_{n}\right) .
\end{aligned}
$$

Then the operator $\Delta$ in (31) is replaced by $\Delta_{1}+\Delta_{\epsilon}+$ $\Delta_{1} \Delta_{\epsilon}$ (see (13) or (15)), and the function $x_{n}$ is replaced by $x(n, \epsilon n)$. The $O \Delta E$ (31) then becomes

$$
\begin{aligned}
\left(\Delta_{1}^{2}+\right. & 2 \Delta_{1}\left(\Delta_{\epsilon}+\Delta_{1} \Delta_{\epsilon}\right)+\Delta_{\epsilon}^{2} \\
& \left.+2 \Delta_{1} \Delta_{\epsilon}^{2}+\Delta_{1}^{2} \Delta_{\epsilon}^{2}\right) x(n, \epsilon n) \\
& +(2-2 \cos (\theta))\left(\Delta_{1}+\Delta_{\epsilon}+\Delta_{1} \Delta_{\epsilon}+I\right) x(n, \epsilon n) \\
= & \epsilon\left(1-\left(\left(\Delta_{1}+\Delta_{\epsilon}+\Delta_{1} \Delta_{\epsilon}+I\right) x(n, \epsilon n)\right)^{2}\right) \\
& \times\left(\left(\Delta_{1}^{2}+2 \Delta_{1}\left(\Delta_{\epsilon}+\Delta_{1} \Delta_{\epsilon}\right)\right.\right. \\
& \left.+\Delta_{\epsilon}^{2}+2 \Delta_{1} \Delta_{\epsilon}^{2}+\Delta_{1}^{2} \Delta_{\epsilon}^{2}\right) x(n, \epsilon n) \\
& \left.+2\left(\Delta_{1}+\Delta_{\epsilon}+\Delta_{1} \Delta_{\epsilon}\right) x(n, \epsilon n)\right) .
\end{aligned}
$$

Then the function $x(n, \epsilon n)$ is expanded in a formal power series in $\epsilon$, that is,

$x(n, \epsilon n)=x_{0}(n, \epsilon n)+\epsilon x_{1}(n, \epsilon n)+\epsilon^{2} x_{2}(n, \epsilon n)+\cdots$,

and is substituted into (32). By taking together those terms of equal powers in $\epsilon$, one obtains as $O$ (1)-problem:

$\Delta_{1}^{2} x_{0}(n, \epsilon n)+(2-2 \cos (\theta))\left(\Delta_{1}+I\right) x_{0}(n, \epsilon n)=0$,

and as $O(\epsilon)$-problem

$$
\begin{aligned}
\epsilon \Delta_{1}^{2} x_{1}(n, \epsilon n)+\epsilon(2-2 \cos (\theta))\left(\Delta_{1}+I\right) x_{1}(n, \epsilon n) \\
=-2 \Delta_{1}\left(\Delta_{\epsilon}+\Delta_{1} \Delta_{\epsilon}\right) x_{0}(n, \epsilon n) \\
\quad-(2-2 \cos (\theta))\left(\Delta_{\epsilon}+\Delta_{1} \Delta_{\epsilon}\right) x_{0}(n, \epsilon n) \\
+\epsilon\left(1-\left(\left(\Delta_{1}+I\right) x_{0}(n, \epsilon n)\right)^{2}\right) \\
\quad \times\left(\left(\Delta_{1}^{2}+2 \Delta_{1}\right) x_{0}(n, \epsilon n)\right),
\end{aligned}
$$

and so on. The $O(1)$-problem (34) can readily be solved, yielding

$x_{0}(n, \epsilon n)=f_{0}(\epsilon n) \cos (n \theta)+g_{0}(\epsilon n) \sin (n \theta)$,

where $f_{0}(\epsilon n)$ and $g_{0}(\epsilon n)$ are still arbitrary functions which can be used to avoid secular terms in $x_{1}(n, \epsilon n)$. Then by substituting (36) into the $O(\epsilon)$-problem (35), and after rearranging terms, one finally obtains as $O(\epsilon)$-problem

$$
\begin{aligned}
& \epsilon \Delta_{1}^{2} x_{1}(n, \epsilon n)+\epsilon(2-2 \cos \theta)\left(\Delta_{1}+I\right) x_{1}(n, \epsilon n) \\
& =\cos (n \theta)\{(1-\cos (2 \theta)) \\
& \times\left(\Delta_{\epsilon} f_{0}(\epsilon n)-\epsilon f_{0}(\epsilon n)\left(1-\frac{1}{4} f_{0}^{2}(\epsilon n)\right.\right. \\
& \left.\left.-\frac{1}{4} g_{0}^{2}(\epsilon n)\right)\right)-\sin (2 \theta)\left(\Delta_{\epsilon} g_{0}(\epsilon n)\right. \\
& \left.\left.-\epsilon g_{0}(\epsilon n)\left(1-\frac{1}{4} f_{0}^{2}(\epsilon n)-\frac{1}{4} g_{0}^{2}(\epsilon n)\right)\right)\right\} \\
& +\sin (n \theta)\left\{\operatorname { s i n } ( 2 \theta ) \left(\Delta_{\epsilon} f_{0}(\epsilon n)-\epsilon f_{0}(\epsilon n)\right.\right. \\
& \left.\times\left(1-\frac{1}{4} f_{0}^{2}(\epsilon n)-\frac{1}{4} g_{0}^{2}(\epsilon n)\right)\right) \\
& +(1-\cos (2 \theta))\left(\Delta_{\epsilon} g_{0}(\epsilon n)-\epsilon g_{0}(\epsilon n)\right. \\
& \left.\left.\times\left(1-\frac{1}{4} f_{0}^{2}(\epsilon n)-\frac{1}{4} g_{0}^{2}(\epsilon n)\right)\right)\right\} \\
& +\epsilon\{\cos (3 n \theta+2 \theta)[(\cos (2 \theta)-1) \\
& \times\left(-\frac{1}{4} f_{0}^{3}(\epsilon n)+\frac{3}{4} f_{0}(\epsilon n) g_{0}^{2}(\epsilon n)\right) \\
& \left.+\sin (2 \theta)\left(-\frac{3}{4} f_{0}^{2}(\epsilon n) g_{0}(\epsilon n)+\frac{1}{4} g_{0}^{3}(\epsilon n)\right)\right] \\
& +\sin (3 n \theta+2 \theta) \\
& \times\left[(\cos (2 \theta)-1)\left(-\frac{3}{4} f_{0}^{2}(\epsilon n) g_{0}(\epsilon n)+\frac{1}{4} g_{0}^{3}(\epsilon)\right)\right. \\
& \left.\left.+\sin (2 \theta)\left(\frac{1}{4} f_{0}^{3}(\epsilon)-\frac{3}{4} f_{0}(\epsilon n) g_{0}^{2}(\epsilon n)\right)\right]\right\} \text {. }
\end{aligned}
$$

In the $O \Delta E$ (37) for $x_{1}(n, \epsilon n)$, it is obvious that the right-hand side contains terms (that is, $\cos (n \theta)$ and 
Fig. 1 The dynamics of the solutions of the $O \Delta E(40)$ for $0<\varepsilon<1$

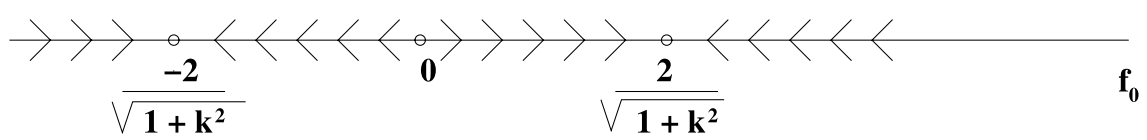

$\sin (n \theta))$, which are solutions of the corresponding homogeneous $O \Delta E$. It should be observed that the terms involving $\cos (3 n \theta+2 \theta)$ and $\sin (3 n \theta+2 \theta)$ are no solutions of the homogeneous $O \Delta E$. So, to avoid unbounded or secular behavior in $x_{1}(n, \epsilon n)$, it follows from (37) that $f_{0}(\epsilon n)$ and $g_{0}(\epsilon n)$ have to satisfy:

$\Delta_{\epsilon} f_{0}(\epsilon n)=\epsilon f_{0}(\epsilon n)\left(1-\frac{1}{4} f_{0}^{2}(\epsilon n)-\frac{1}{4} g_{0}^{2}(\epsilon n)\right)$,
$\Delta_{\epsilon} g_{0}(\epsilon n)=\epsilon g_{0}(\epsilon n)\left(1-\frac{1}{4} f_{0}^{2}(\epsilon n)-\frac{1}{4} g_{0}^{2}(\epsilon n)\right)$.

From (38), it is obvious that when $f_{0}(\epsilon n)$ (or $g_{0}(\epsilon n)$ ) is equal to zero for some $n=n_{0}$, then $f_{0}(\epsilon n)$ (or $\left.g_{0}(\epsilon n)\right)$ is zero for all $n \geq n_{0}$. It also follows from (38) that $g_{0}(\epsilon n) \Delta_{\epsilon} f_{0}(\epsilon n)-f_{0}(\epsilon n) \Delta_{\epsilon} g_{0}(\epsilon n)=0$ or equivalently (assuming that $f_{0}(\epsilon n) \neq 0$ ):

$\Delta\left(\frac{g_{0}(\epsilon n)}{f_{0}(\epsilon n)}\right)=0 \quad \Leftrightarrow \quad g_{0}(\epsilon n)=k f_{0}(\epsilon, n)$

for some constant $k$, which is determined by the initial conditions. The system of two first order $O \Delta E$ s (38) then reduces by using (39) to the following first order $O \Delta E$ for $f_{0}(\epsilon n)$.

$\Delta_{\epsilon} f_{0}(\epsilon n)=\epsilon f_{0}(\epsilon n)\left(1-\frac{1}{4}\left(1+k^{2}\right) f_{0}^{2}(\epsilon n)\right)$.

As far as we know, there are no exact solutions available for the $O \Delta E$ (40). However, for $0<\epsilon<1$, the $O \Delta E$ (40) has three equilibrium points: an unstable one for $f_{0}=0$, and two stable ones: one for $f_{0}=\frac{-2}{\sqrt{1+k^{2}}}$ and one for $f_{0}=\frac{2}{\sqrt{1+k^{2}}}$. The dynamics of the solutions of the $O \Delta E$ (40) is depicted in Fig. 1.

For $\epsilon \geq 1$, the dynamics becomes much more complicated (three unstable equilibrium points, period doublings, chaotic behavior), but since $0<$ $\epsilon \ll 1$, that case is beyond the scope of the analysis. From (39), (40), and Fig. 1, it now follows that (for $\left.f_{0}^{2}(\epsilon n)+g_{0}^{2}(\epsilon n) \neq 0\right) f_{0}^{2}(\epsilon n)+g_{0}^{2}(\epsilon n)$ tends (slowly) to 4 for $n \rightarrow \infty$. From (37), $x_{1}(n, \epsilon n)$ can now be determined such that $x_{1}(n, \epsilon n)$ is bounded for $n \sim \frac{1}{\epsilon}$, and from (36) and (39), it follows that

$x_{0}(n, \epsilon n)=f_{0}(\epsilon n) \sqrt{1+k^{2}} \sin (n \theta+\varphi)$, where $\varphi$ is given by $\sin (\varphi)=\frac{1}{\sqrt{1+k^{2}}}$ and $\cos (\varphi)=$ $\frac{k}{\sqrt{1+k^{2}}}$, and where $f_{0}(\epsilon n)$ is a solution of the $O \Delta E$ (40) (see also Fig. 1). So far, an approximation $x_{\text {approx }}(n, \epsilon n)=x_{0}(n, \epsilon n)+\epsilon x_{1}(n, \epsilon n)$ has been constructed, where $x_{1}(n, \epsilon n)$ still contains some arbitrary functions which can be used to avoid secular terms in $x_{2}(n, \epsilon n)$. Since we are not interested in the higher order approximations, there arbitrary functions will or can be chosen equal to their initial values. The approximation $x_{\text {approx }}(n, \epsilon n)$ satisfied the $O \Delta E$ (30) accurately, that is, up to $O\left(\epsilon^{2}\right)$. In fact, it can be shown that

$$
\begin{aligned}
& x_{\text {approx }}(n+2, \epsilon(n+2)) \\
& \quad-2 \cos (\theta) x_{\text {approx }}(n+1, \epsilon(n+1)) \\
& +x_{\text {approx }}(n, \epsilon n) \\
& -\epsilon\left(1-x_{\text {approx }}^{2}(n+1, \epsilon(n+1))\right) \\
& \quad \times\left(x_{\text {approx }}(n+2, \epsilon(n+2))\right. \\
& \left.-x_{\text {approx }}(n, \epsilon n)\right)=\epsilon^{2} R(n, \epsilon n),
\end{aligned}
$$

where $R(n, \epsilon n)$ depends on $x_{0}(n, \epsilon n)$ and on $x_{1}(n, \epsilon n)$, and where $R(n, \epsilon n)$ is bounded for $n \sim \frac{1}{\epsilon}$. It requires an additional analysis to show that $x_{\text {approx }}(n, \epsilon n)$ and $x_{0}(n, \epsilon n)$ are both $O(\epsilon)$ accurate approximations of the solution of (30) for $n \sim \frac{1}{\epsilon}$. The proof of asymptotic validity of the approximation(s) on long iteration scales (that is, for $n \sim \frac{1}{\epsilon}$ ) will be given in the next section of this paper.

\section{On the asymptotic validity of approximations}

In this section, a justification of the multiple scales perturbation method for $O \Delta E$ s will be given, which covers all the examples that have been presented in this paper. Consider the following system of $k$ first order $O \Delta E \mathrm{~s}$ :

$\underline{u}(n+1)=A \underline{u}(n)+\epsilon \underline{f}(\underline{u}(n), n ; \epsilon)$,

where $\underline{u}(n)$ is a $(k \times 1)$-vector, $\mathrm{A}$ is a $(k \times k)$-matrix with constant and $\epsilon$-independent elements, $\epsilon$ is a small 
parameter with $0<\epsilon \ll 1$, and where $\underline{f}$ is $(k \times 1)$ vector, with $\underline{f}: \mathbb{R}^{k} \times \mathbb{R} \rightarrow \mathbb{R}^{k}$. Moreover, $\underline{f}$ satisfies the following Lipschitz condition, that is,

$$
\|\underline{f}(\underline{u}(n), n ; \epsilon)-\underline{f}(\underline{\tilde{u}}(n), n ; \epsilon)\| \leq L\|\underline{u}(n)-\underline{\tilde{u}}(n)\|
$$

for some constant $L$, and where $\underline{\tilde{u}}(n)$ is a $(k \times 1)$ vector, and where $\|$.$\| is assumed to be the maximum$ norm. The vector function $f$ is not necessarily linear in $\underline{u}(n)$. Let $\underline{\tilde{u}}(n)$ be an approximation of $\underline{u}(n)$, which has been constructed by some kind of perturbation method (for instance, a multiple scales perturbation method or another perturbation method), and let $\underline{\tilde{u}}(n)$ satisfy

$\underline{\tilde{u}}(n+1)=A \underline{\tilde{u}}(n)+\epsilon \underline{f}(\underline{\tilde{u}}(n), n ; \epsilon)+\epsilon^{m+1} \underline{R}(n ; \epsilon)$,

where $\underline{R}(n ; \epsilon)$ is a $(k \times 1)$-vector, and where $m$ is a positive integer (usually $m=1$ ). Now it will be assumed that matrix $A$ is similar to a diagonal matrix, that is, it will be assumed that there exists a nonsingular matrix $P$ (with $\|P\|$ and $\left\|P^{-1}\right\|$ bounded by a constant $M_{0}$ ) such that $P^{-1} A P$ is a diagonal matrix with on the diagonal the eigenvalue $\lambda_{1}, \lambda_{2}, \ldots, \lambda_{k}$ of matrix $A$. When $A$ is not similar to a diagonal matrix (that is, when the algebraic multiplicity of an eigenvalue is not equal to the geometric multiplicity of this eigenvalue), then a similar proof can be given by using the Jordan form of matrix $A$. This almost similar proof will be omitted in this paper. Now let $\underline{u}(n)=P \underline{v}(n)$ and $\tilde{u}(n)=P \underline{\tilde{v}}(n)$. Then (43) and (45) become

$$
\begin{aligned}
\underline{v}(n+1)= & P^{-1} A P \underline{v}(n)+\epsilon P^{-1} \underline{f}(P \underline{v}(n), n ; \epsilon), \\
\underline{\tilde{v}}(n+1)= & P^{-1} A P \underline{\tilde{v}}(n)+\epsilon P^{-1} \underline{f}(P \underline{\tilde{v}}(n), n ; \epsilon) \\
& +\epsilon^{m+1} P^{-1} \underline{R}(n ; \epsilon),
\end{aligned}
$$

where $P^{-1} A P$ is a diagonal matrix with on the diagonal the eigenvalues $\lambda_{1}, \ldots, \lambda_{k}$ of matrix $A$. Now let

$$
\lambda=\max _{1 \leq i \leq k}\left|\lambda_{i}\right|
$$

If $\lambda \leq 1$, it will be assumed that for $n \sim \frac{1}{\epsilon}$

$$
\|\underline{R}(n ; \epsilon)\| \leq M_{1},
$$

where $M_{1}$ is a positive constant. And for $\lambda>1$, it will be assumed that for $n \sim \frac{1}{\epsilon}$

$$
\begin{aligned}
& \|\underline{R}(n ; \epsilon)\| \leq M_{2} \lambda^{n}\left(1+M_{3} \epsilon\right)^{n}, \quad \text { and } \\
& \|\underline{f}(\underline{u}(n), n ; \epsilon)\| \\
& \quad \leq M_{4}\|\underline{u}(n)\|+M_{5} \text { for }\|\underline{u}(n)\| \rightarrow \infty,
\end{aligned}
$$

where $M_{2}, M_{3}, M_{4}$, and $M_{5}$ are positive constants. Condition (50) for $\lambda>1$ indicates that the linear part in (43) dominates the dynamics of the problem (or equivalently, the term $\epsilon f$ in (43) remains relatively small compared to the other terms in (43)). Now first, the case $0<\lambda \leq 1$ will be considered. The degenerate case $\lambda=0$ will not be considered in this paper. By subtracting the two equations in (46), and by using (44), (47), and (48), the following estimate can be obtained.

$$
\begin{aligned}
& \|\underline{v}(n+1)-\underline{\tilde{v}}(n+1)\| \\
& \quad \leq\left(\lambda+\epsilon L M_{0}\right)\|\underline{v}(n)-\underline{\tilde{v}}(n)\|+\epsilon^{m+1} M_{0} M_{1} \Rightarrow \\
& \|\underline{v}(n)-\underline{v}(n)\| \\
& \quad \leq\left(\lambda+\epsilon L M_{0}\right)^{n}\|\underline{v}(0)-\underline{\tilde{v}}(0)\| \\
& \quad+\epsilon^{m+1} M_{0} M_{1}\left(\frac{\left(\lambda+\epsilon L M_{0}\right)^{n}-1}{\lambda+\epsilon L M_{0}-1}\right) .
\end{aligned}
$$

From (51), it follows that for $0<\lambda<1$

$$
\begin{aligned}
\|\underline{v}(n)-\underline{\tilde{v}}(n)\| \leq & \|\underline{v}(0)-\underline{\tilde{v}}(0)\| O\left(\lambda^{n} e^{\epsilon n \frac{L M_{0}}{\lambda}}\right) \\
& +O\left(\epsilon^{m+1} \lambda^{n} e^{\epsilon n \frac{L M_{0}}{\lambda}}\right),
\end{aligned}
$$

and for $\lambda=1$ that

$$
\begin{aligned}
& \|\underline{v}(n)-\underline{\tilde{v}}(n)\| \\
& \quad \leq\|\underline{v}(0)-\underline{\tilde{v}}(0)\| O\left(e^{\epsilon n L M_{0}}\right)+O\left(\epsilon^{m} e^{\epsilon n L M_{0}}\right) .
\end{aligned}
$$

Now if $\|\underline{u}(0)-\underline{\tilde{u}}(0)\|=O\left(\epsilon^{m+1}\right)$ for $0<\lambda<1$, and if $\|\underline{u}(0)-\underline{\tilde{u}}(0)\|=O\left(\epsilon^{m}\right)$, for $\lambda=1$, it follows from (52) that for $0<\lambda<1$

$$
\begin{aligned}
\|\underline{u}(n)-\underline{\tilde{u}}(n)\| & =\left\|P^{-1} \underline{v}(n)-P^{-1} \underline{\tilde{v}}(n)\right\| \\
& \leq M_{0}\|\underline{v}(n)-\underline{\tilde{v}}(n)\|=O\left(\epsilon^{m+1} \lambda^{n}\right)
\end{aligned}
$$

for $n=O\left(\frac{1}{\epsilon}\right)$, and it follows from (53) that for $\lambda=1$

$\|\underline{u}(n)-\underline{\tilde{u}}(n)\|=O\left(\epsilon^{m}\right)$

for $n=O\left(\frac{1}{\epsilon}\right)$. 
Now the case $\lambda>1$ will be considered. Again, consider (46) and let $\underline{v}(n)=\lambda^{n} \underline{w}(n)$ and $\underline{\tilde{v}}(n)=\lambda^{n} \underline{\tilde{w}}(n)$. System (46) then becomes

$$
\begin{aligned}
\underline{w}(n+1)= & \frac{1}{\lambda} P^{-1} A P \underline{w}(n) \\
& +\frac{\epsilon}{\lambda^{n+1}} P^{-1} \underline{f}\left(\lambda^{n} P \underline{w}(n), n ; \epsilon\right), \\
\underline{\tilde{w}}(n+1)= & \frac{1}{\lambda} P^{-1} A P \underline{\tilde{w}}(n) \\
& +\frac{\epsilon}{\lambda^{n+1}} P^{-1} \underline{f}\left(\lambda^{n} P \underline{\tilde{w}}(n), n ; \epsilon\right) \\
& +\frac{\epsilon^{m+1}}{\lambda^{n+1}} P^{-1} \underline{R}(n ; \epsilon) .
\end{aligned}
$$

In (56), it should be observed that $\frac{1}{\lambda} P^{-1} A P$ is a diagonal matrix for which the modulus of the largest diagonal element in modules is equal to one. Then by subtracting the two equations in (56), and by using (44), (47), (49), and (50), it follows that (assuming that $\left.\|\underline{w}(0)-\underline{\tilde{w}}(0)\|=O\left(\epsilon^{m}\right)\right)$

$$
\begin{aligned}
& \|\underline{w}(n+1)-\underline{\tilde{w}}(n+1)\| \\
& \leq 1 .\|\underline{w}(n)-\underline{\tilde{w}}(n)\|+\frac{\epsilon L M_{0}}{\lambda}\|\underline{w}(n)-\underline{\tilde{w}}(n)\| \\
& \quad+\frac{\epsilon^{m+1}}{\lambda^{n+1}} M_{0}\|\underline{R}(n ; \epsilon)\| \Rightarrow \\
& \|\underline{w}(n)-\underline{\tilde{w}}(n)\| \\
& \leq \quad\left(1+\frac{\epsilon L M_{0}}{\lambda}\right)^{n} \\
& \quad \times\left\{\|\underline{w}(0)-\underline{\tilde{w}}(0)\|+O\left(\epsilon^{m} e^{\epsilon n M_{3}}\right)\right\} \Rightarrow \\
& \|\underline{w}(n)-\underline{\tilde{w}}(n)\|=O\left(\epsilon^{m}\right) \quad \text { for } n \sim \frac{1}{\epsilon} .
\end{aligned}
$$

From $\underline{u}(n)=P \underline{v}(n)=\lambda^{n} P \underline{w}(n)$ and $\underline{\tilde{u}}(n)=$ $\lambda^{n} P \underline{\tilde{w}}(n)$, and from (57), it can then be deduced that for $\lambda>1$

$\|\underline{u}(n)-\underline{\tilde{u}}(n)\|=O\left(\epsilon^{m} \lambda^{n}\right)$

for $n=O\left(\frac{1}{\epsilon}\right)$. The results which have been proved so far are summarized in the following theorem.

Theorem 4.1 Let $\underline{u}(n)$ and $\underline{\tilde{u}}(n)$ satisfy (43) and (45), respectively, where $f$ satisfies (44) and where matrix $A$ is assumed to be similar to a diagonal matrix. Let $\lambda$ be defined by (47), and let $\underline{R}$ and $f$ additionally satisfy (48) or (49), (50) for $0<\lambda \leq 1$ or $\lambda>1$, respectively. Furthermore, let $\|\underline{u}(0)-\underline{\tilde{u}}(0)\|$ be
$O\left(\epsilon^{m+1}\right)$ for $0<\lambda<1$ and $O\left(\epsilon^{m}\right)$ for $\lambda \geq 1$. Then for $n=O\left(\frac{1}{\epsilon}\right)$, it follows that

$\|\underline{u}(n)-\underline{\tilde{u}}(n)\|=O\left(\epsilon^{m+1} \lambda^{n}\right) \quad$ for $0<\lambda<1$,

and

$\|\underline{u}(n)-\underline{\tilde{u}}(n)\|=O\left(\epsilon^{m} \lambda^{n}\right) \quad$ for $\lambda \geq 1$.

Since each $k$-th order $O \Delta E$ can be rewritten as a system of $k$ first order $O \Delta E \mathrm{~s}$, it follows that Theorem 4.1 directly can be applied to the examples as treated in the previous sections (see the linear $O \Delta E$ (16) and the weakly nonlinear $O \Delta E$ (30)). For both examples, it can be simply shown that $\lambda=1$ and that $\left|x_{n}-\left(x_{0}(n, \epsilon n)+\epsilon x_{1}(n, \epsilon n)\right)\right|=O(\epsilon)$ for $n=O\left(\frac{1}{\epsilon}\right)$. It also follows for $n=O\left(\frac{1}{\epsilon}\right)$ that

$$
\begin{aligned}
& \left|x_{n}-x_{0}(n, \epsilon n)\right| \\
& \quad=\left|x_{n}-\left(x_{0}(n, \epsilon n)+\epsilon x_{1}(n, \epsilon n)\right)+\epsilon x_{1}(n, \epsilon n)\right| \\
& \quad \leq\left|x_{n}-\left(x_{0}(n, \epsilon n)+\epsilon x_{1}(n, \epsilon n)\right)\right|+\epsilon\left|x_{1}(n, \epsilon n)\right| \\
& \quad=O(\epsilon)+O(\epsilon)=O(\epsilon)
\end{aligned}
$$

since $x_{1}(n, \epsilon n)$ is bounded for $n=O\left(\frac{1}{\epsilon}\right)$. So, in both examples, the functions $x_{0}(n, \epsilon n)$ are $O(\epsilon)$ accurate approximation of $x_{n}$ for $0 \leq n \leq \frac{K}{\epsilon}$, where $K$ is an $\epsilon$-independent constant.

\section{On singularly perturbed, linear $O \Delta E s$}

In this section, the following three singularly perturbed, linear, second order $O \Delta E$ s will be studied for $n=0,1,2, \ldots$ :

$\left(\epsilon-\frac{1}{4}\right) x_{n+2}+\left(\frac{1}{2}-2 \epsilon\right) x_{n+1}+\epsilon x_{n}=0$,

$(\epsilon+2) y_{n+2}-(1+2 \epsilon) y_{n+1}+\epsilon y_{n}=0, \quad$ and

$z_{n+2}+\epsilon z_{n+1}+\epsilon z_{n}=0$,

where $\epsilon$ is a small parameter with $0<\epsilon \ll 1$. A singularly perturbed $O \Delta E$ is characterized by the fact that the order of the $O \Delta E$ is reduced when the small parameter $\epsilon$ is taken equal to zero (in this case). In the $O \Delta E \mathrm{~s}(59)$ and (60), the order of the $O \Delta E$ s will be reduced by one, whereas for $O \Delta E \mathrm{~s}$ (61) with $\epsilon=0$, one can hardly speak of an $O \Delta E$. When a naive, straightforward perturbation approach is used to approximate the solution of the $O \Delta E$, it is usually impossible to 
satisfy all the initial conditions because of this order reduction in the $O \Delta E$. For $O D E \mathrm{~s}$, rescaling procedures are used to tackle this problem. In this section, a rescaling procedure for $O \Delta E$ s like (59)-(61) will be proposed to solve the aforementioned problem for $O \Delta E \mathrm{~s}$. When the significant scalings in the $O \Delta E \mathrm{~s}$ are known, the multiple scales perturbation method for $O \Delta E$ s (as developed in Sect. 2 of this paper) will be applied to approximate the solutions of the $O \Delta E \mathrm{~s}$. In fact, a two scales method will be used for (59), and a three scales method will be used for (60), and for (61). It will turn out that the constructed approximations of the solutions are accurate ones on long iteration scales. This can be shown by using the theory as developed in the previous section or by comparing the approximations directly with the exact solutions. Now the $O \Delta E$ (59) will be considered firstly.

\subsection{The $O \Delta E(59)$}

To determine the significant scalings in (59), the following rescaling procedure is proposed. Let

$x_{n}=\delta_{n}(\epsilon) a_{n}$,

where $\delta_{n}(\epsilon)$ is a rescaling function which might depend on $\epsilon$, and where $a_{n}$ is a function which remains $O(1)$ on the iteration scale under consideration (usually this will be an iteration scale of $O\left(\frac{1}{\epsilon}\right)$ ). Then (62) is substituted into (59), yielding

$$
\begin{aligned}
& \left(\epsilon-\frac{1}{4}\right) \delta_{n+2}(\epsilon) a_{n+2}+\left(\frac{1}{2}-2 \epsilon\right) \delta_{n+1}(\epsilon) a_{n+1} \\
& \quad+\epsilon \delta_{n}(\epsilon) a_{n}=0
\end{aligned}
$$

and a balancing procedure will be followed to determine the rescaling function(s) $\delta_{n}(\epsilon)$. When the first term and the second term in (63) are assumed to be the most significant ones, then $\delta_{n}(\epsilon)$ has to satisfy

$\frac{1}{4} \delta_{n+2}(\epsilon)+\frac{1}{2} \delta_{n+1}(\epsilon)=0 \Rightarrow \delta_{n}(\epsilon)=\delta_{0} 2^{n}$.

It can readily be verified that the third term in (63) (that is, $\epsilon \delta_{n}(\epsilon) a_{n}$ ) is indeed smaller. So, $\delta(\epsilon)=2^{n}$ is indeed a significant rescaling. When the second term and the third term in (63) are assumed to be the most significant ones, then $\delta_{n}(\epsilon)$ has to satisfy

$\frac{1}{2} \delta_{n+1}(\epsilon)+\epsilon \delta_{n}(\epsilon)=0 \Rightarrow \delta_{n}(\epsilon)=\delta_{0}(-2 \epsilon)^{n}$.

It can be verified that the first term in (63) is indeed smaller, and so, $\delta_{n}(\epsilon)=(-2 \epsilon)^{n}$ is another significant rescaling. Similarly, it can be checked that the first term and the third term in (63) cannot be the most significant ones simultaneously, since the second term would be in that case larger. Now two functionally independent approximations of the solutions of the linear $O \Delta E$ (59) will be constructed. The sum of these two approximations will be an approximation of the general solution of the $O \Delta E$ (59). Firstly, let $x_{n}=2^{n} a_{n}$. The $O \Delta E$ (59) then becomes

$(-1+4 \epsilon) a_{n+2}+(1-4 \epsilon) a_{n+1}+\epsilon a_{n}=0$.

A two scales perturbation method will be used to approximate the solution $a_{n}$ of (66) since the straightforward perturbation expansion will lead to secular terms. The following expansion for $a_{n}$

$$
\begin{aligned}
a_{n} & =a(n, \epsilon n) \\
& =a_{0}(n, \epsilon n)+\epsilon a_{1}(n, \epsilon n)+\epsilon^{2} a_{2}(n, \epsilon n)+\cdots
\end{aligned}
$$

is substituted into (66), and terms of equal powers in $\epsilon$ are taken together (see also Sect. 2 of this paper), yielding as $O(1)$-problem

$$
\begin{aligned}
& \Delta_{1}^{2} a_{0}(n, \epsilon n)+\Delta_{1} a_{0}(n, \epsilon n)=0 \\
& \quad \Leftrightarrow \quad a_{0}(n+2, \epsilon n)-a_{0}(n+1, \epsilon n)=0,
\end{aligned}
$$

and as $O(\epsilon)$-problem

$$
\begin{aligned}
\epsilon \Delta_{1}^{2} a_{1}(n, \epsilon n)+\epsilon \Delta_{1} a_{1}(n, \epsilon n) \\
=-2 \Delta_{1}\left(\Delta_{1} \Delta_{\epsilon}+\Delta_{\epsilon}\right) a_{0}(n, \epsilon n) \\
\quad+4 \epsilon \Delta_{1}^{2} a_{0}(n, \epsilon n)-\left(\Delta_{1} \Delta_{\epsilon}+\Delta_{\epsilon}\right) a_{0}(n, \epsilon n) \\
\quad+4 \epsilon \Delta_{1} a_{0}(n, \epsilon n)+\epsilon x_{0}(n, \epsilon n),
\end{aligned}
$$

and so on. The $O(1)$-problem (68) can readily be solved, yielding

$a_{0}(n, \epsilon n)=f_{0}(\epsilon n)$, 
where $f_{0}(\epsilon n)$ is an arbitrary function which will be used to avoid secular terms in $a_{1}(n, \epsilon n)$. The $O(\epsilon)$ problem (69) then becomes

$$
\begin{gathered}
\epsilon \Delta_{1}^{2} a_{1}(n, \epsilon n)+\epsilon \Delta_{1} a_{1}(n, \epsilon n) \\
=-\Delta_{\epsilon} f_{0}(\epsilon n)+\epsilon f_{0}(\epsilon n) .
\end{gathered}
$$

To avoid secular terms in $a_{1}(n, \epsilon n)$, it is obvious from (71) that $f_{0}(\epsilon n)$ should satisfy

$$
-\Delta_{\epsilon} f_{0}(\epsilon n)+\epsilon f_{0}(\epsilon n)=0 \Rightarrow f_{0}(\epsilon n)=c_{0}(1+\epsilon)^{n},
$$

and

$a_{1}(n, \epsilon n)=f_{1}(\epsilon n)$,

where $c_{0}$ is an arbitrary constant, and where $f_{1}(\epsilon n)$ is an arbitrary function which can be used to avoid secular terms in $a_{2}(n, \epsilon n)$. Since we are not interested in the higher order approximation, $f_{1}(\epsilon n)$ will now be taken equal to its initial value $c_{1}$. So far, the following approximation for $a_{n}$ has been constructed (see (67), (70), and (72)): $c_{0}(1+\epsilon)^{n}+\epsilon c_{1}$, where $c_{0}$ and $c_{1}$ are constants. And so, it follows that an approximation of a solution of (59) is

$2^{n}\left(c_{0}(1+\epsilon)^{n}+\epsilon c_{1}\right)$.

Another functionally independent approximation of the solution of (59) can be constructed by using the rescaling given by (65), that is, let $x_{n}=(-2 \epsilon)^{n} b_{n}$. The $O \Delta E(59)$ then becomes

$\epsilon(1-4 \epsilon) b_{n+2}+(1-4 \epsilon) b_{n+1}-b_{n}=0$.

The following expansion for $b_{n}$

$$
\begin{aligned}
b_{n} & =b(n, \epsilon n) \\
& =b_{0}(n, \epsilon n)+\epsilon b_{1}(n, \epsilon n)+\epsilon^{2} b_{2}(n, \epsilon n)+\cdots
\end{aligned}
$$

is substituted into (74), and terms of equal powers in $\epsilon$ are taken together, yielding as $O(1)$-problem

$\Delta_{1} b_{0}(n, \epsilon n)=0$,

and as $O(\epsilon)$-problem

$$
\begin{aligned}
& \epsilon \Delta_{1} b_{1}(n, \epsilon n) \\
& =-\epsilon \Delta_{1}^{2} b_{0}(n, \epsilon n)-\left(\Delta_{1} \Delta_{\epsilon}+\Delta_{\epsilon}\right) b_{0}(n, \epsilon n) \\
& \quad+2 \epsilon \Delta_{1} b_{0}(n, \epsilon n)+3 \epsilon b_{0}(n, \epsilon n),
\end{aligned}
$$

and so on. The $O(1)$-problem (76) can readily be solved, yielding

$b_{0}(n, \epsilon n)=g_{0}(\epsilon n)$,

where $g_{0}(\epsilon n)$ is an arbitrary function which will be used to avoid secular terms in $b_{1}(n, \epsilon n)$. The $O(\epsilon)$ (77) now becomes

$\epsilon \Delta_{1} b_{1}(n, \epsilon n)=-\Delta_{\epsilon} g_{0}(\epsilon n)+3 \epsilon g_{0}(\epsilon n)$.

To avoid secular terms in $b_{1}(n, \epsilon n)$ it is clear from (79) that $g_{0}(\epsilon n)$ should satisfy

$$
\begin{gathered}
-\Delta_{\epsilon} g_{0}(\epsilon n)+3 \epsilon g_{0}(\epsilon n)=0 \\
\Rightarrow \quad g_{0}(\epsilon n)=d_{0}(1+3 \epsilon)^{n},
\end{gathered}
$$

and

$b_{1}(n, \epsilon n)=g_{1}(\epsilon n)$,

where $d_{0}$ is an arbitrary constant, and where $g_{1}(\epsilon n)$ is an arbitrary function which can be used to avoid secular terms in $b_{2}(n, \epsilon n)$. Since we are not interested in the higher order approximations, $g_{1}(\epsilon n)$ will now be taken equal to a constant $d_{1}$. So far, the following approximation for $b_{n}$ has been constructed (see (75), (78), and (80)): $d_{0}(1+3 \epsilon)^{n}+\epsilon d_{1}$, where $d_{0}$ and $d_{1}$ are constants. And so, it follows that an approximation of a solution of (59) is

$(-2 \epsilon)^{n}\left(d_{0}(1+3 \epsilon)^{n}+\epsilon d_{1}\right)$.

Since the $O \Delta E(59)$ is linear, the superposition principle can be used, and then it follows from (73) and (81) that an approximation $x(n, \epsilon n)$ of the solution $x_{n}$ of (59) is given by

$$
\begin{aligned}
x(n, \epsilon n)= & 2^{n}\left(c_{0}(1+\epsilon)^{n}+\epsilon c_{1}\right) \\
& +(-2 \epsilon)^{n}\left(d_{0}(1+3 \epsilon)^{n}+\epsilon d_{1}\right),
\end{aligned}
$$

where $c_{0}, c_{1}, d_{0}$, and $d_{1}$ are constants which are determined by the initial conditions for $n=0,1$ (that is, when $x_{0}$ and $x_{1}$, and their expansions in $\epsilon$ are given). How well $x(n, \epsilon n)$ approximates the exact solution $x_{n}$ can now be determined in two ways. The first way is to apply Theorem 4.1 (see the previous section). For $O \Delta E(59)$, we have that $\lambda=2$ and $x(n, \epsilon n)$ satisfies the $O \Delta E$ (59) up to $(2+2 \epsilon)^{n} O\left(\epsilon^{2}\right)+(-2 \epsilon-$ $6 \epsilon)^{n} O\left(\epsilon^{3}\right)$. So, it follows from Theorem 4.1 that

$$
\left|x_{n}-x(n, \epsilon n)\right|=2^{n} O(\epsilon) \text { for } n=O\left(\frac{1}{\epsilon}\right) \text {. }
$$


From (83), it follows that the absolute error becomes large on the iteration scale of $O\left(\frac{1}{\epsilon}\right)$, but that the relative error is small for $n \sim \frac{1}{\epsilon}$, that is,

$\left|\frac{x_{n}-x(n, \epsilon n)}{x(n, \epsilon n)}\right|=O(\epsilon) \quad$ for $n \sim \frac{1}{\epsilon}$.

Since the linear $O \Delta E$ (59) has constant coefficients also the exact solution $x_{n}$ can be computed directly, yielding

$$
\begin{aligned}
x_{n}= & a\left(1+(1-4 \epsilon)^{-\frac{1}{2}}\right)^{n}+b\left(1-(1-4 \epsilon)^{-\frac{1}{2}}\right)^{n} \\
= & a\left(2+2 \epsilon+6 \epsilon^{2}+O\left(\epsilon^{3}\right)\right)^{n} \\
& +b\left(-2 \epsilon-6 \epsilon^{2}+O\left(\epsilon^{3}\right)\right)^{n}
\end{aligned}
$$

where $a$ and $b$ are constants. It can also readily be verified from (85) that $x(n, \epsilon n)$ satisfies (83) and (84).

\subsection{The $O \Delta E(60)$}

The rescaling and balancing procedure as introduced in Sect. 5.1 can be repeated for the $O \Delta E$ (60), and it turns out that the significant scalings are

$\delta_{n}(\epsilon)=\left(\frac{1}{2}\right)^{n}, \quad$ and $\quad \delta_{n}(\epsilon)=\epsilon^{n}$.

As in the previous example, two functionally independent approximations of the solutions of the linear $O \Delta E$ (60) will be constructed, but in this example by using a three scales perturbation method instead of a two scales method. By putting $y_{n}=\left(\frac{1}{2}\right)^{n} a_{n}$, the $O \Delta E$ (60) becomes

$(\epsilon+2) a_{n+2}-(2+4 \epsilon) a_{n+1}+4 \epsilon a_{n}=0$.

Then the following expansion for $a_{n}$

$$
\begin{aligned}
a_{n}= & a_{0}\left(n, \epsilon n, \epsilon^{2} n\right)+\epsilon a_{1}\left(n, \epsilon n, \epsilon^{2} n\right) \\
& +\epsilon^{2} a_{2}\left(n, \epsilon n, \epsilon^{2} n\right)+\cdots
\end{aligned}
$$

is substituted into (87), and as usual the following $O\left(\epsilon^{m}\right)$-problems are obtained for $m=0,1,2, \ldots$ (see also Sect. 2 of this paper): the $O$ (1)-problem:

$$
\begin{aligned}
& 2 \Delta_{1}^{2} a_{0}\left(n, \epsilon n, \epsilon^{2} n\right)+2 \Delta_{1} a_{0}\left(n, \epsilon n, \epsilon^{2} n\right)=0 \\
& \quad \Leftrightarrow \quad a_{0}\left(n+2, \epsilon n, \epsilon^{2} n\right)-a_{0}\left(n+1, \epsilon n, \epsilon^{2} n\right)=0,
\end{aligned}
$$

the $O(\epsilon)$-problem:

$$
\begin{aligned}
2 \epsilon \Delta_{1}^{2} & a_{1}\left(n, \epsilon n, \epsilon^{2} n\right)+2 \Delta_{1} a_{1}\left(n, \epsilon n, \epsilon^{2} n\right) \\
= & -\left(4 \Delta_{1}+2\right)\left(\Delta_{1} \Delta_{\epsilon}+\Delta_{\epsilon}\right) a_{0}\left(n, \epsilon n, \epsilon^{2} n\right) \\
& -\epsilon \Delta_{1}^{2} a_{0}\left(n, \epsilon n, \epsilon^{2} n\right)+2 \epsilon \Delta_{1} a_{0}\left(n, \epsilon n, \epsilon^{2} n\right) \\
& -\epsilon a_{0}\left(n, \epsilon n, \epsilon^{2} n\right),
\end{aligned}
$$

the $O\left(\epsilon^{2}\right)$-problem:

$$
\begin{aligned}
2 \epsilon^{2} \Delta & a_{2}\left(n, \epsilon n, \epsilon^{2} n\right)+2 \epsilon^{2} \Delta_{1} a_{2}\left(n, \epsilon n, \epsilon^{2} n\right) \\
= & -\epsilon\left(4 \Delta_{1}+2\right)\left(\Delta_{1} \Delta_{\epsilon}+\Delta_{\epsilon}\right) a_{1}\left(n, \epsilon n, \epsilon^{2} n\right) \\
& -\epsilon^{2} \Delta_{1}^{2} a_{1}\left(n, \epsilon n, \epsilon^{2} n\right)+2 \epsilon^{2} \Delta_{1} a_{1}\left(n, \epsilon n, \epsilon^{2} n\right) \\
& -\epsilon^{2} a_{1}\left(n, \epsilon n, \epsilon^{2} n\right)-\left(4 \Delta_{1}\left(\Delta_{1} \Delta_{\epsilon^{2}}+\Delta_{\epsilon^{2}}\right)\right. \\
& \left.+2\left(\Delta_{1} \Delta_{\epsilon}+\Delta_{\epsilon}\right)^{2}\right) a_{0}\left(n, \epsilon n, \epsilon^{2} n\right) \\
& -\epsilon\left(2 \Delta_{1}-2\right)\left(\Delta_{1} \Delta_{\epsilon}+\Delta_{\epsilon}\right) a_{0}\left(n, \epsilon n, \epsilon^{2} n\right) \\
& -2\left(\Delta_{1} \Delta_{\epsilon^{2}}+\Delta_{\epsilon^{2}}\right) a_{0}\left(n, \epsilon n, \epsilon^{2} n\right),
\end{aligned}
$$

and so on. The $O(1)$-problem (89) readily can be solved, yielding

$a_{0}\left(n, \epsilon n, \epsilon^{2} n\right)=f_{0}\left(\epsilon n, \epsilon^{2} n\right)$,

where $f_{0}\left(\epsilon n, \epsilon^{2} n\right)$ is still an arbitrary function which will be used to avoid secular terms in $a_{1}\left(n, \epsilon n, \epsilon^{2} n\right)$ and in $a_{2}\left(n, \epsilon n, \epsilon^{2} n\right)$. The $O(\epsilon)$-problem (90) now becomes

$$
\begin{aligned}
2 \epsilon & \Delta_{1}^{2} a_{1}\left(n, \epsilon n, \epsilon^{2} n\right)+2 \epsilon \Delta_{1} a_{1}\left(n, \epsilon n, \epsilon^{2} n\right) \\
& =-2 \Delta_{\epsilon} f_{0}\left(\epsilon n, \epsilon^{2} n\right)-\epsilon f_{0}\left(\epsilon n, \epsilon^{2} n\right) .
\end{aligned}
$$

To avoid secular terms in $a_{1}\left(n, \epsilon n, \epsilon^{2} n\right)$, it is obvious from (93) that $f_{0}\left(\epsilon n, \epsilon^{2} n\right)$ has to satisfy

$$
\begin{gathered}
-2 \Delta_{\epsilon} f_{0}\left(\epsilon n, \epsilon^{2} n\right)-\epsilon f_{0}\left(\epsilon n, \epsilon^{2} n\right)=0 \\
\Rightarrow \quad f_{0}\left(\epsilon n, \epsilon^{2} n\right)=\left(1-\frac{\epsilon}{2}\right)^{n} g_{0}\left(\epsilon^{2} n\right),
\end{gathered}
$$

where $g_{0}\left(\epsilon^{2} n\right)$ is still an arbitrary function which will be used to avoid secular terms in the $O\left(\epsilon^{2}\right)$-problem (91). From (93) and (94), it now also follows that

$a_{1}\left(n, \epsilon n, \epsilon^{2} n\right)=f_{1}\left(\epsilon n, \epsilon^{2} n\right)$,

where $f_{1}\left(\epsilon n, \epsilon^{2} n\right)$ is still an arbitrary function which can be used to avoid secular terms in the higher order problems. The $O\left(\epsilon^{2}\right)$-problem (91) now becomes 
(observe that $\Delta_{1} a_{0}=0$ and $\Delta_{1} a_{1}=0$ ):

$$
\begin{aligned}
2 \epsilon^{2} \Delta_{1} & a_{2}\left(n, \epsilon n, \epsilon^{2} n\right)+2 \epsilon^{2} \Delta_{1} a_{2}\left(n, \epsilon n, \epsilon^{2} n\right) \\
= & -2 \epsilon \Delta_{\epsilon} f_{1}\left(\epsilon n, \epsilon^{2} n\right)-\epsilon^{2} f_{1}\left(\epsilon n, \epsilon^{2} n\right) \\
& -2 \Delta_{\epsilon}^{2} f_{0}\left(\epsilon n, \epsilon^{2} n\right)-2 \Delta_{\epsilon^{2}} f_{0}\left(\epsilon n, \epsilon^{2} n\right) \\
& +2 \epsilon \Delta_{\epsilon} f_{0}\left(\epsilon n, \epsilon^{2} n\right) .
\end{aligned}
$$

Now it should be observed that all terms in the right-hand side of (96) will lead to secular terms in $a_{2}\left(n, \epsilon n, \epsilon^{2} n\right)$. To avoid these secular terms, the righthand side of (96) should be equal to zero, or equivalently (by using (94)):

$$
\begin{aligned}
2 \epsilon & \Delta_{\epsilon} f_{1}\left(\epsilon n, \epsilon^{2} n\right)+\epsilon^{2} f_{1}\left(\epsilon n, \epsilon^{2} n\right) \\
& =\left(1-\frac{\epsilon}{2}\right)^{n}\left(-2 \Delta_{\epsilon^{2}} g_{0}\left(\epsilon^{2} n\right)-\frac{3}{2} \epsilon^{2} g_{0}\left(\epsilon^{2} n\right)\right) .
\end{aligned}
$$

Now all terms in the right-hand side of (97) will lead to secular terms in $f_{1}\left(\epsilon n, \epsilon^{2} n\right)$. To avoid this secular behavior, it follows from (97) that $g_{0}\left(\epsilon^{2} n\right)$ has to satisfy:

$$
\begin{gathered}
-2 \Delta_{\epsilon^{2}} g_{0}\left(\epsilon^{2} n\right)-\frac{3}{2} \epsilon^{2} g_{0}\left(\epsilon^{2} n\right)=0 \\
\Rightarrow g_{0}\left(\epsilon^{2} n\right)=c_{0}\left(1-\frac{3}{4} \epsilon^{2}\right)^{n},
\end{gathered}
$$

where $c_{0}$ is a constant. From (97) it then also follows that

$f_{1}\left(\epsilon n, \epsilon^{2} n\right)=\left(1-\frac{\epsilon}{2}\right)^{n} g_{1}\left(\epsilon^{2} n\right)$,

where $g_{1}\left(\epsilon^{2} n\right)$ is an arbitrary function. And from (96), it then follows that

$a_{2}\left(n, \epsilon n, \epsilon^{2} n\right)=f_{2}\left(\epsilon n, \epsilon^{2} n\right)$,

where $f_{2}\left(\epsilon n, \epsilon^{2} n\right)$ is an arbitrary function. From $y_{n}=$ $\left(\frac{1}{2}\right)^{n} a_{n},(88),(92),(94)-(100)$, it follows that an approximation of $y_{n}$ is given by

$c_{0}\left(\frac{1}{2}\right)^{n}\left(1-\frac{\epsilon}{2}\right)^{n}\left(1-\frac{3}{4} \epsilon^{2}\right)^{n}+O(\epsilon)\left(\frac{1}{2}\right)^{n}$.

In a completely similar way a second, functionally independent approximation of $y_{n}$ can be constructed (starting with $y_{n}=\epsilon^{n} b_{n}$, and so on), yielding

$d_{0} \epsilon^{n}\left(1+\epsilon^{2}\right)^{n}+\epsilon^{n} O(\epsilon)$,

where $d_{0}$ is a constant. The computations to obtain (102) are left to the reader as an exercise. From (101) and (102), it follows by using the superposition principle that an approximation $y\left(n, \epsilon n, \epsilon^{2} n\right)$ of the general solution $y_{n}$ of (60) is given by

$$
\begin{aligned}
& y\left(n, \epsilon n, \epsilon^{2} n\right) \\
& \quad=c_{0}\left(\frac{1}{2}\right)^{n}\left(1-\frac{\epsilon}{2}\right)^{n}\left(1-\frac{3}{4} \epsilon^{2}\right)^{n}+d_{0} \epsilon^{n}\left(1+\epsilon^{2}\right)^{n} .
\end{aligned}
$$

How accurate this approximation is, can directly be seen by comparing $y\left(n, \epsilon n, \epsilon^{2} n\right)$ with the exact solution $y_{n}$ which is given by

$$
\begin{aligned}
y_{n}= & a\left(\frac{1+2 \epsilon+(1-4 \epsilon)^{\frac{1}{2}}}{2(\epsilon+2)}\right)^{n} \\
& +b\left(\frac{1+2 \epsilon-(1-4 \epsilon)^{\frac{1}{2}}}{2(\epsilon+2)}\right)^{n} \\
= & a\left(\frac{1}{2}-\frac{1}{4} \epsilon-\frac{3}{8} \epsilon^{2}+O\left(\epsilon^{3}\right)\right)^{n} \\
& +b\left(\epsilon+\epsilon^{3}+O\left(\epsilon^{4}\right)\right)^{n} .
\end{aligned}
$$

From (103) and (104), it can readily be deduced that for $n \sim \frac{1}{\epsilon^{2}}$

$$
\left|y_{n}-y\left(n, \epsilon n, \epsilon^{2} n\right)\right|=\left(\frac{1}{2}\right)^{n} O(\epsilon) .
$$

\subsection{The $O \Delta E(61)$}

The rescaling and balancing procedure as introduced in Sect. 5.1 can be repeated again for the $O \Delta E(61)$, and it turns out that only one significant scaling is present:

$\delta_{n}(\epsilon)=(\sqrt{\epsilon})^{n}$.

As in the previous examples, approximations of the solution of the linear $O \Delta E$ (61) will be constructed. In this example, a three scales perturbation method will be applied. By putting $z_{n}=(\sqrt{\epsilon})^{n} a_{n}$, the $O \Delta E$ (61) becomes

$a_{n+2}+\sqrt{\epsilon} a_{n+1}+a_{n}=0$. 
Then the following expansion for $a_{n}$

$$
\begin{aligned}
a_{n}= & a_{0}(n, \sqrt{\epsilon} n, \epsilon n)+\sqrt{\epsilon} a_{1}(n, \sqrt{\epsilon} n, \epsilon n) \\
& +\epsilon a_{2}(n, \sqrt{\epsilon} n, \epsilon n)+\cdots
\end{aligned}
$$

is substituted into (106), and as usual the following $O\left(\epsilon^{\frac{m}{2}}\right)$-problems are obtained for $m=0,1,2, \ldots$ : the $O(1)$-problem:

$$
\begin{aligned}
& \Delta_{1}^{2} a_{0}(n, \sqrt{\epsilon} n, \epsilon n)+2 \Delta_{1} a_{0}(n, \sqrt{\epsilon} n, \epsilon n) \\
& \quad+2 a_{0}(n, \sqrt{\epsilon} n, \epsilon n)=0 \Leftrightarrow \\
& a_{0}(n+2, \sqrt{\epsilon} n, \epsilon n)+a_{0}(n, \sqrt{\epsilon} n, \epsilon n)=0,
\end{aligned}
$$

the $O(\sqrt{\epsilon})$-problem:

$$
\begin{aligned}
\sqrt{\epsilon} \Delta_{1}^{2} a_{1}(n, \sqrt{\epsilon} n, \epsilon n)+2 \sqrt{\epsilon} \Delta_{1} a_{1}(n, \sqrt{\epsilon} n, \epsilon n) \\
+2 \sqrt{\epsilon} a_{1}(n, \sqrt{\epsilon} n, \epsilon n) \\
=-2\left(\Delta_{1}+I\right)\left(\Delta_{1} \Delta_{\sqrt{\epsilon}}+\Delta_{\sqrt{\epsilon}}\right) a_{0}(n, \sqrt{\epsilon} n, \epsilon n) \\
\quad-\sqrt{\epsilon}\left(\Delta_{1}+I\right) a_{0}(n, \sqrt{\epsilon} n, \epsilon n)
\end{aligned}
$$

the $O(\epsilon)$-problem:

$$
\begin{aligned}
\epsilon \Delta_{1}^{2} a_{2}(n, \sqrt{\epsilon} n, \epsilon n)+2 \epsilon \Delta_{1} a_{2}(n, \sqrt{\epsilon} n, \epsilon n) \\
+2 \epsilon a_{2}(n, \sqrt{\epsilon} n, \epsilon n) \\
=-\sqrt{\epsilon}\left(2 \Delta_{\sqrt{\epsilon}} E_{1}^{2}+\sqrt{\epsilon} E_{1}\right) a_{1}(n, \sqrt{\epsilon} n, \epsilon n) \\
-\left(2 \Delta_{3} E_{1}^{2}+\Delta_{\sqrt{\epsilon}}^{2} E_{1}^{2}+\sqrt{\epsilon} \Delta_{\sqrt{\epsilon}} E_{1}\right) \\
\times a_{0}(n, \sqrt{\epsilon} n, \epsilon n),
\end{aligned}
$$

and so on. The $O(1)$-problem (108) can easily be solved, yielding

$$
\begin{aligned}
& a_{0}(n, \sqrt{\epsilon} n, \epsilon n) \\
& \quad=f_{0}(\sqrt{\epsilon} n, \epsilon n) \cos \left(\frac{n \pi}{2}\right)+g_{0}(\sqrt{\epsilon} n, \epsilon n) \sin \left(\frac{n \pi}{2}\right),
\end{aligned}
$$

where $f_{0}(\sqrt{\epsilon} n, \epsilon n)$ and $g_{0}(\sqrt{\epsilon} n, \epsilon n)$ are still arbitrary functions which will be used to avoid secular terms in $a_{1}(n, \sqrt{\epsilon} n, \epsilon n)$ and in $a_{2}(n, \sqrt{\epsilon} n, \epsilon n)$. The
$O(\sqrt{\epsilon})$-problem (110) now becomes

$$
\begin{aligned}
\sqrt{\epsilon} \Delta_{1}^{2} a_{1}(n, \sqrt{\epsilon} n, \epsilon n)+2 \sqrt{\epsilon} \Delta_{1} a_{1}(n, \sqrt{\epsilon} n, \epsilon n) \\
+2 \sqrt{\epsilon} a_{1}(n, \sqrt{\epsilon} n, \epsilon n) \\
=\cos \left(\frac{n \pi}{2}\right)\left(2 \Delta_{\sqrt{\epsilon}} f_{0}(\sqrt{\epsilon} n, \epsilon n)-\sqrt{\epsilon} g_{0}(\sqrt{\epsilon} n, \epsilon n)\right) \\
+\sin \left(\frac{n \pi}{2}\right)\left(2 \Delta_{\sqrt{\epsilon}} g_{0}(\sqrt{\epsilon} n, \epsilon n)\right. \\
\left.+\sqrt{\epsilon} f_{0}(\sqrt{\epsilon} n, \epsilon n)\right) .
\end{aligned}
$$

Obviously, the right-hand side of (112) contains terms (i.e., $\cos \left(\frac{n \pi}{2}\right)$ and $\sin \left(\frac{n \pi}{2}\right)$ ) which are solutions of the corresponding homogeneous $O \Delta E$ and which consequently lead to secular terms in $a_{1}(n, \sqrt{\epsilon} n, \epsilon n)$. To avoid this secular behavior in $a_{1}(n, \sqrt{\epsilon} n, \epsilon n)$, it follows from (112) that $f_{0}(\sqrt{\epsilon} n, \epsilon n)$ and $g_{0}(\sqrt{\epsilon} n, \epsilon n)$ have to satisfy

$$
\begin{aligned}
& 2 \Delta_{\sqrt{\epsilon}} f_{0}(\sqrt{\epsilon} n, \epsilon n)-\sqrt{\epsilon} g_{0}(\sqrt{\epsilon} n, \epsilon n)=0, \\
& 2 \Delta_{\sqrt{\epsilon}} g_{0}(\sqrt{\epsilon} n, \epsilon n)+\sqrt{\epsilon} f_{0}(\sqrt{\epsilon} n, \epsilon n)=0 .
\end{aligned}
$$

System (113) can readily be solved, yielding

$$
\begin{aligned}
f_{0}(\sqrt{\epsilon} n, \epsilon n)= & \alpha_{0}(\epsilon n)\left(1+\frac{\epsilon}{4}\right)^{\frac{n}{2}} \cos (n \mu(\epsilon)) \\
& +\beta_{0}(\epsilon n)\left(1+\frac{\epsilon}{4}\right)^{\frac{n}{2}} \sin (n \mu(\epsilon)), \\
g_{0}(\sqrt{\epsilon} n, \epsilon n)= & -\alpha_{0}(\epsilon n)\left(1+\frac{\epsilon}{4}\right)^{\frac{n}{2}} \sin (n \mu(\epsilon)) \\
& +\beta_{0}(\epsilon n)\left(1+\frac{\epsilon}{4}\right)^{\frac{n}{2}} \cos (n \mu(\epsilon)),
\end{aligned}
$$

where $\alpha_{0}(\epsilon n)$ and $\beta_{0}(\epsilon n)$ are arbitrary functions which will be used to avoid secular terms in $a_{2}(n, \sqrt{\epsilon} n, \epsilon n)$, and where $\mu(\epsilon)$ is given by

$$
\cos (\mu(\epsilon))=\left(1+\frac{\epsilon}{4}\right)^{-\frac{1}{2}},
$$

and

$$
\sin (\mu(\epsilon))=\frac{1}{2} \sqrt{\epsilon}\left(1+\frac{\epsilon}{4}\right)^{-\frac{1}{2}},
$$

and

$\mu(\epsilon)=\frac{1}{2} \sqrt{\epsilon}-\frac{1}{24} \epsilon \sqrt{\epsilon}+O\left(\epsilon^{2} \sqrt{\epsilon}\right)$ for $\epsilon \rightarrow 0$. 
Then it follows from (112) that $a_{1}(n, \sqrt{\epsilon} n, \epsilon n)$ is given by

$$
\begin{aligned}
& a_{1}(n, \sqrt{\epsilon} n, \epsilon n) \\
& \quad=f_{1}(\sqrt{\epsilon} n, \epsilon n) \cos \left(\frac{n \pi}{2}\right)+g_{1}(\sqrt{\epsilon} n, \epsilon n) \sin \left(\frac{n \pi}{2}\right),
\end{aligned}
$$

where $f_{1}(\sqrt{\epsilon} n, \epsilon n)$ and $g_{1}(\sqrt{\epsilon} n, \epsilon n)$ are arbitrary functions which can be used to avoid secular terms in the solutions of the higher order problems. By using (111), (114), and (116), it follows that the $O(\epsilon)$ problem (110) now becomes

$$
\begin{aligned}
\epsilon \Delta_{1}^{2} a_{2} & (n, \sqrt{\epsilon} n, \epsilon n)+2 \epsilon \Delta_{1} a_{2}(n, \sqrt{\epsilon} n, \epsilon n) \\
& +2 \epsilon a_{2}(n, \sqrt{\epsilon} n, \epsilon n) \\
= & \cos \left(\frac{n \pi}{2}\right)\left\{2 \sqrt{\epsilon} \Delta_{\sqrt{\epsilon}} f_{1}(\sqrt{\epsilon} n, \epsilon n)\right. \\
& -\epsilon g_{1}(\sqrt{\epsilon} n, \epsilon n) \\
& \left.+2 \Delta_{\epsilon} f_{0}(\sqrt{\epsilon} n, \epsilon n)+\frac{\epsilon}{4} f_{0}(\sqrt{\epsilon} n, \epsilon n)\right\} \\
& +\sin \left(\frac{n \pi}{2}\right)\left\{2 \sqrt{\epsilon} \Delta_{\sqrt{\epsilon}} g_{1}(\sqrt{\epsilon} n, \epsilon n)\right. \\
& +\epsilon f_{1}(\sqrt{\epsilon} n, \epsilon n)+2 \Delta_{\epsilon} g_{0}(\sqrt{\epsilon} n, \epsilon n) \\
+ & \left.\frac{\epsilon}{4} g_{0}(\sqrt{\epsilon} n, \epsilon n)\right\} .
\end{aligned}
$$

To avoid secular terms in $a_{2}(n, \sqrt{\epsilon} n, \epsilon n)$, it is obvious from (117) that $f_{1}, g_{1}, f_{0}$, and $g_{0}$ have to satisfy

$$
\begin{aligned}
& 2 \sqrt{\epsilon} \Delta_{\sqrt{\epsilon}} f_{1}(\sqrt{\epsilon} n, \epsilon n)-\epsilon g_{1}(\sqrt{\epsilon} n, \epsilon n) \\
&=-\left(2 \Delta_{\epsilon} \alpha_{0}(\epsilon n)+\frac{\epsilon}{4} \alpha_{0}(\epsilon n)\right)\left(1+\frac{\epsilon}{4}\right)^{\frac{n}{2}} \cos (n \mu(\epsilon)) \\
&-\left(2 \Delta_{\epsilon} \beta_{0}(\epsilon n)+\frac{\epsilon}{4} \beta_{0}(\epsilon n)\right)\left(1+\frac{\epsilon}{4}\right)^{\frac{n}{2}} \sin (n \mu(\epsilon)), \\
& 2 \sqrt{\epsilon} \Delta_{\sqrt{\epsilon}} g_{1}(\sqrt{\epsilon} n, \epsilon n)+\epsilon g_{1}(\sqrt{\epsilon} n, \epsilon n) \\
&=\left(2 \Delta_{\epsilon} \alpha_{0}(\epsilon n)+\frac{\epsilon}{4} \alpha_{0}(\epsilon n)\right)\left(1+\frac{\epsilon}{4}\right)^{\frac{n}{2}} \sin (n \mu(\epsilon)) \\
&-\left(2 \Delta_{\epsilon} \beta_{0}(\epsilon n)+\frac{\epsilon}{4} \beta_{0}(\epsilon n)\right)\left(1+\frac{\epsilon}{4}\right)^{\frac{n}{2}} \\
& \times \cos (n \mu(\epsilon)) .
\end{aligned}
$$

Since $\left(1+\frac{\epsilon}{4}\right)^{\frac{n}{2}} \cos (n \mu(\epsilon))$, and $\left(1+\frac{\epsilon}{4}\right)^{\frac{n}{2}} \sin (n \mu(\epsilon))$ are solutions of the homogeneous system (118), it is obvious that these terms lead to secular behavior in $f_{1}(\sqrt{\epsilon} n, \epsilon n)$, and in $g_{1}(\sqrt{\epsilon} n, \epsilon n)$. To avoid this secular behavior, it follows from (118) that $\alpha_{0}(\epsilon n)$ and $\beta_{0}(\epsilon n)$ have to satisfy

$2 \Delta_{\epsilon} \alpha_{0}(\epsilon n)+\frac{\epsilon}{4} \alpha_{0}(\epsilon n)=0$,
$2 \Delta_{\epsilon} \beta_{0}(\epsilon n)+\frac{\epsilon}{4} \beta_{0}(\epsilon n)=0$.

System (119) can readily be solved, yielding

$$
\begin{aligned}
& \alpha_{0}(\epsilon n)=k_{0}\left(1-\frac{\epsilon}{8}\right)^{n}, \\
& \beta_{0}(\epsilon n)=l_{0}\left(1-\frac{\epsilon}{8}\right)^{n},
\end{aligned}
$$

where $k_{0}$ and $l_{0}$ are arbitrary constants. From (118), $f_{1}$ and $g_{1}$ can now be determined, and from (117) $a_{2}$ can be determined, yielding

$$
\begin{aligned}
f_{1}(\sqrt{\epsilon} n, \epsilon n)= & \alpha_{1}(\epsilon n)\left(1+\frac{\epsilon}{4}\right)^{\frac{n}{2}} \cos (n \mu(\epsilon)) \\
& +\beta_{1}(\epsilon n)\left(1+\frac{\epsilon}{4}\right)^{\frac{n}{2}} \sin (n \mu(\epsilon)), \\
g_{1}(\sqrt{\epsilon} n, \epsilon n)= & -\alpha_{1}(\epsilon n)\left(1+\frac{\epsilon}{4}\right)^{\frac{n}{2}} \sin (n \mu(\epsilon)) \\
& +\beta_{1}(\epsilon n)\left(1+\frac{\epsilon}{4}\right)^{\frac{n}{2}} \cos (n \mu(\epsilon)), \\
a_{2}(n, \sqrt{\epsilon} n, \epsilon n)= & f_{2}(\sqrt{\epsilon} n, \epsilon n) \cos \left(\frac{n \pi}{2}\right) \\
& +g_{2}(\sqrt{\epsilon} n, \epsilon n) \sin \left(\frac{n \pi}{2}\right),
\end{aligned}
$$

where $\alpha_{1}, \beta_{1}, f_{2}$, and $g_{2}$ are arbitrary functions. To determine these functions completely, the $O(\epsilon \sqrt{\epsilon})$ problem and the $O\left(\epsilon^{2}\right)$-problem have to be solved. Since we are not interested in the higher order approximations at this moment, we will take $\alpha_{1}, \beta_{1}, f_{2}$, and $g_{2}$ equal to constants (such that the initial conditions (if present) can be satisfied up to $O(\epsilon)$ ). From (111), (114), (115), and (120), it follows that $a_{0}(n, \sqrt{\epsilon} n, \epsilon n)$ 
is given by

$$
\begin{aligned}
& a_{0}(n, \sqrt{\epsilon} n, \epsilon n) \\
& =k_{0}\left(1-\frac{\epsilon}{8}\right)^{n}\left(1+\frac{\epsilon}{4}\right)^{\frac{n}{2}} \cos \left(\frac{n \pi}{2}+n \mu(\epsilon)\right) \\
& \quad+l_{0}\left(1-\frac{\epsilon}{8}\right)^{n}\left(1+\frac{\epsilon}{4}\right)^{\frac{n}{2}} \sin \left(\frac{n \pi}{2}+n \mu(\epsilon)\right),
\end{aligned}
$$

where $k_{0}$ and $l_{0}$ are constants. The exact solution of the $O \Delta E(106)$ is given by

$a_{n}=k 1^{n} \cos (n \theta(\epsilon))+l 1^{n} \sin (n \theta(\epsilon))$,

where $k$ and $l$ are constants, and where $\theta(\epsilon)$ is given by

$$
\cos (\theta(\epsilon))=-\frac{1}{2} \sqrt{\epsilon}
$$

and

$$
\sin (\theta(\epsilon))=\left(1-\frac{\epsilon}{4}\right)^{\frac{1}{2}},
$$

and

$\theta(\epsilon)=\frac{\pi}{2}+\frac{1}{2} \sqrt{\epsilon}+\frac{1}{48} \epsilon \sqrt{\epsilon}+O\left(\epsilon^{2} \sqrt{\epsilon}\right)$ for $\epsilon \rightarrow 0$.

From (121) and (122), it is not difficult to deduce that

$\left|a_{n}-a_{0}(n, \sqrt{\epsilon} n, \epsilon n)\right|=O(\sqrt{\epsilon})$ for $n=O\left(\frac{1}{\epsilon}\right)$.

The examples as presented in this section and in Sects. 2 and 3 of this paper clearly prove that the multiple scales perturbation method gives accurate approximations on long iteration scales.

\section{Conclusions and remarks}

In this paper, a version of the multiple scales perturbation method for $O \Delta E$ s has been presented and formulated completely in terms of difference equations. It has been shown how this method can be applied to regularly perturbed $O \Delta E$ s and to singularly perturbed, linear $O \Delta E$ s. The relative and/or absolute errors in the constructed approximations of the solutions of the $O \Delta E$ s have been determined, and it has been shown that these approximations are valid on long iteration scales.
It is to be expected that the presented perturbation method also can be applied successfully to weakly perturbed partial difference equations, and to singularly perturbed, weakly nonlinear $O \Delta E$ s. Of course, these extensions will be interesting subjects for future research. Finally, it should be remarked that the presented perturbation method also can be used in the numerical analysis of certain classes of regularly or singularly perturbed differential equations to see whether the solutions of the discretized equations (i.e., the difference equations) have the same type of behavior as the solutions of the differential equations or not.

Acknowledgements Part of the research presented in this paper was performed by the first author as a visiting professor at the Department of Applied Mathematics of the University of Washington in Seattle during the spring quarter of 2007. The hospitality of the members of the department and the many discussions with Professor R.E. O'Malley, Jr. on mathematics, in general, and on differential and difference equations, in particular, are highly appreciated.

Open Access This article is distributed under the terms of the Creative Commons Attribution Noncommercial License which permits any noncommercial use, distribution, and reproduction in any medium, provided the original author(s) and source are credited.

\section{References}

1. Agarwal, R.P.: Difference Equations and Inequalities: Theory, Methods, and Applications. Dekker, New York (1992)

2. Agarwal, R., Bohner, M., O’Regan, D., Peterson, A.: Dynamic equations on time scales: a survey. J. Comput. Appl. Math. 141, 1-26 (2002)

3. Andrianov, I.V., Manevitch, L.I.: Asymptotology: Ideas, Methods, and Applications. Kluwer Academic, Dordrecht (2002)

4. Elaydi, S.: An Introduction to Difference Equations, 3rd edn. Springer, New York (2005)

5. Holmes, M.H.: Introduction to Perturbation Methods. Springer, New York (1995)

6. Hoppensteadt, F.C., Miranker, W.L.: Multitime methods for systems of difference equations. Stud. Appl. Math. 56, 273-289 (1977)

7. Huston, R.L.: Krylov-Bogoljubov method for difference equations. SIAM J. Appl. Math. 19, 334-339 (1979)

8. Kadalbajoo, M.K., Patidar, K.C.: A survey of numerical techniques for solving singularly perturbed ordinary differential equations. Appl. Math. Comput. 130, 457-510 (2002)

9. Kelly, W.G., Peterson, A.C.: Difference Equations, An Introduction with Applications. Academic Press, New York (1991)

10. Kevorkian, J., Cole, J.D.: Multiple Scale and Singular Perturbation Methods. Springer, New York (1996) 
11. Kosevich, A.M.: The Crystal Lattice: Phonons, Solitons, Dislocations. Wiley-VCH, New York (1999)

12. Kosevich, A.M., Kovalev, A.S.: Self-localization of vibrations in a one-dimensional anharmonic chain. Sov. Phys. JETP 40, 891-896 (1975)

13. Luongo, A.: Perturbation methods for nonlinear autonomous discrete-time dynamical systems. Nonlinear Dyn. 10, 317-331 (1996)

14. Maccari, A.: A perturbation method for nonlinear twodimensional maps. Nonlinear Dyn. 19, 295-312 (1999)

15. Marathe, A., Chatterjee, A.: Wave attenuation in nonlinear periodic structures using harmonic balance and multiple scales. J. Sound Vib. 289, 871-888 (2006)

16. Mickens, R.E.: Difference Equations. Reinhold, New York (1987)

17. Mickens, R.E.: Periodic solutions of second order nonlinear difference equations containing a small parameter-IV. Multi-discrete time method. J. Franklin Inst. 324, 263-271 (1987)
18. Murdock, J.A.: Perturbations, Theory and Methods. Wiley, New York (1991)

19. O'Malley, R.E., Jr.: Singular Perturbation Methods for Ordinary Differential Equations. Springer, New York (1991)

20. Nayfeh, A.H.: Perturbation Methods. Wiley-Interscience, New York (1973)

21. Sari, T., Zerizer, T.: Perturbations for linear difference equations. J. Math. Anal. Appl. 305, 43-52 (2005)

22. Subramanian, R., Krishnan, A.: Non-linear discrete time systems analysis by multiple time perturbation techniques. J. Sound Vib. 63, 325-335 (1979)

23. Torng, H.C.: Second order non-linear difference equations containing small parameters. J. Franklin Inst. 269, 97-104 (1960)

24. Verhulst, F.: Methods and Applications of Singular Perturbations. Springer, New York (2005) 